\title{
HYDRODYNAMIC EQUATIONS FOR INCOMPRESSIBLE INVISCID FLUID IN TERMS OF GENERALIZED STREAM FUNCTION
}

\author{
YURI A. RYLOV
}

Received 5 August 2003

\begin{abstract}
Hydrodynamic equations for ideal incompressible fluid are written in terms of generalized stream function. Two-dimensional version of these equations is transformed to the form of one dynamic equation for the stream function. This equation contains arbitrary function which is determined by inflow conditions given on the boundary. To determine unique solution, velocity and vorticity (but not only velocity itself) must be given on the boundary. This unexpected circumstance may be interpreted in the sense that the fluid has more degrees of freedom than it was believed. Besides, the vorticity is a less observable quantity as compared with the velocity. It is shown that the Clebsch potentials are used essentially at the description of vortical flow.
\end{abstract}

2000 Mathematics Subject Classification: 76M99, 35J65.

1. Introduction. In this paper, we write hydrodynamic equations for the ideal fluid in terms of Clebsch potentials [4, 5]. This representation admits one to integrate hydrodynamic equations and to obtain effective description of rotational stream flow. We have discovered an unexpected fact that the effective description of the rotational flow cannot be carried out without introduction of the Clebsch potentials. In general, the problem of irrotational stream flow and that of rotational stream flow are stated differently. We show this in an example of stationary two-dimensional flow of incompressible fluid. In this case, one of Clebsch potentials may coincide with the stream function $\psi$. The irrotational flow is described by the equation for stream function $\psi$ :

$$
\psi_{x x}+\psi_{y y}=0
$$

where indices mean corresponding partial derivatives. For the rotational stationary twodimensional flow, (1.1) transforms to the form

$$
\psi_{x x}+\psi_{y y}=\Omega(\psi)
$$

where $\Omega(\psi)$ is some function of argument $\psi$ describing the character and intensity of vorticity. The obtained equation is distinguished from (1.1) in an additional term. Both (1.1) and (1.2) are the elliptic type equations. To obtain a unique solution of (1.1), it is sufficient to give value of the stream function $\psi$ on the boundary $\Sigma$ of the volume $V$, where the flow is considered. The same is valid for (1.2), provided that the form of the function $\Omega(\psi)$ is known. But the value of $\psi$ on $\Sigma$ does not admit one to determine the form of the function $\Omega(\psi)$. For determination of $\Omega(\psi)$, one needs some additional information given on the boundary $\Sigma$. 
To state the problem of the rotational stream flow, it is necessary to determine what kind of information on the boundary is sufficient for the derivation of the unique solution of (1.2). Theory of rotational flows cannot exist without statement of this problem. Unfortunately, we have not seen in literature a statement of the problem of the rotational stream flow; we know only single exact solutions [14]. It means that the theory of rotational flows does not exist.

Equation (1.1) is a special case of (1.2) when $\Omega(\psi)=0$. Equation (1.2) is obtained from the conventional hydrodynamic equations for two-dimensional stationary flow of incompressible fluid, which have the form

$$
u_{x}+v_{y}=0, \quad \partial_{y}\left(u u_{x}+v u_{y}\right)=\partial_{x}\left(u v_{x}+v v_{y}\right)
$$

where $u$ and $v$ are velocity components along $x$-axis and $y$-axis, respectively. Introducing the stream function $\psi$,

$$
u=-\psi_{y}, \quad v=\psi_{x}
$$

we satisfy the first equation in (1.3) identically. The second equation in (1.3) takes the form

$$
\frac{\partial(\omega, \psi)}{\partial(x, y)}=0, \quad \omega \equiv \psi_{x x}+\psi_{y y}
$$

Relation (1.2) is the general solution $\omega=\Omega(\psi)$ of (1.5), where $\Omega$ is an arbitrary function of $\psi$.

The goal of the present paper is the statement of the problem of the rotational stream flow. The problem is solved effectively in terms of the generalized stream function (GSF) which has several components. GSF is a generalization of the usual stream function $\psi$ for two-dimensional flow onto a more complicated $n$-dimensional case. Unfortunately, in other more complicated cases, the statement of the problem of the rotational flow needs a special well-developed technique.

This technique is based on application of Jacobian technique to the descriptions of hydrodynamic equations written in terms of Clebsch potentials. We will refer to this technique as the GSF technique. Use of Jacobian technique in application to Clebsch potentials goes back to papers by Clebsch [4,5], who obtained his potentials dealing with Jacobians. In contemporary hydrodynamics, the Clebsch potentials are considered formally without connection with the Jacobian technique [12]. Clebsch potentials have also other names (Lagrangian coordinates, Lagrangian variables [20], and labels of fluid particles). There are several versions of representation of Clebsch potentials. Elements of Jacobian technique were used by many authors (see $[1,8,11,18,20]$ and many others). But in solution of the problem of streamline flow, the Jacobian technique is not used practically, and so are Clebsch potentials. We are interested only in that version, which is connected with a use of Jacobian technique (GSF technique). We use spacetime symmetric version of the Jacobian technique which appears to be simple and effective. It seems that the progress in the investigation of vortical flows is connected mainly with the developed Jacobian technique used together with Clebsch potentials 
(GSF technique). Presentation of the GSF technique can be found in [15], as well as in [16], where it is used for description of the fluid flow in terms of the wave functions.

Why are Clebsch potentials important in the statement of the problem on vortical stream flow? The term $\Omega(\psi)$ in the right-hand side of (1.2) describes vorticity. According to Kelvin's theorem on circulation, the vorticity is "frozen in the fluid," and vorticity travels together with the fluid. Clebsch potentials (labels of the fluid particles), as well as the GSF are also frozen in the fluid. They also travel together with the fluid and vorticity. As a result, the Clebsch potentials (labels) and the GSF appear to be an effective tool in the vorticity description.

A rotational fluid flow has some properties which are absent in irrotational flows.

(1) Consideration of the fluid displacement and a use of Clebsch potentials describing this displacement is essential in rotational flows because this displacement transfers vorticity, which influences the velocity field, whereas such a transport is of no importance in irrotational flows, where the vorticity vanishes.

(2) The boundary conditions for a rotational flow contain more information than the boundary conditions for irrotational flow. This additional information is information on vorticity, which conserves in any ideal fluid. Although the field of vorticity

$$
\boldsymbol{\omega}=\nabla \times \mathbf{v}
$$

is determined by the velocity field $\mathbf{v}$, it is valid only inside some 3 -volume $V$, where vorticity can be determined as a result of differentiation of the velocity field. On the boundary $\Sigma$ of 3-volume $V$, one can calculate only the component of $\boldsymbol{\omega}$ along the normal to $\Sigma$. Components of vorticity $\boldsymbol{\omega}$ tangent to $\Sigma$ must be given additionally. It means that additional (as compared with the irrotational flow) degrees of freedom appear in the rotational flow, and additional information in boundary conditions is necessary for their description.

The first property is analyzed in [15]. We present it here also. It is a common practice to think that the problem of streamline flow can be solved by consideration of only Euler system of equations

$$
\begin{gathered}
\frac{\partial \rho}{\partial t}+\nabla(\rho \mathbf{v})=0, \\
\frac{\partial \mathbf{v}}{\partial t}+(\mathbf{v} \nabla) \mathbf{v}=-\frac{1}{\rho} \nabla p, \quad p=\rho^{2} \frac{\partial E}{\partial \rho}, \\
\frac{\partial S}{\partial t}+(\mathbf{v} \nabla) S=0,
\end{gathered}
$$

where $p$ is the pressure and $E=E(\rho, S)$ is the internal energy of a unit mass considered to be a function of the mass density $\rho$ and the entropy $S$. The internal energy $E=E(\rho, S)$ is a unique characteristic of the ideal fluid. Displacement of the fluid particles (i.e., their trajectories and the law of motion along them) in the given velocity field $\mathbf{v}$ is described by the equations

$$
\frac{d \mathbf{x}}{d t}=\mathbf{v}(t, \mathbf{x}), \quad \mathbf{x}=\mathbf{x}(t, \boldsymbol{\xi})
$$


where $\mathbf{v}(t, \mathbf{x})$ is a solution of the system (1.7), (1.8), and (1.9). Equations (1.7), (1.8), (1.9), and (1.10) form the complete system of hydrodynamic equations, but the form of this system is not quite consistent because dynamic variables $\rho$, $\mathbf{v}$, and $S$ in (1.7), (1.8), and (1.9) are functions of $t, \mathbf{x}$, whereas $\mathbf{x}$ in (1.10) is a function of $t$ and of the label $\boldsymbol{\xi}$ of the fluid particle.

Let $\boldsymbol{\xi}=\boldsymbol{\xi}(t, \mathbf{x})=\left\{\xi_{\alpha}(t, \mathbf{x})\right\}, \alpha=1,2,3$, be three independent integrals of (1.10). Values $\boldsymbol{\xi}$ of three integrals can label fluid particles (Lagrangian coordinates). Then labels $\boldsymbol{\xi}$ satisfy three equations

$$
\frac{\partial \boldsymbol{\xi}}{\partial t}+(\mathbf{v} \nabla) \boldsymbol{\xi}=0
$$

which are equivalent to three equations (1.10). The system of equations (1.7), (1.8), (1.9), and (1.10) is hardly perceived as a whole dynamic system because the system (1.7), (1.8), and (1.9) is closed and its dynamic variables $\rho, \mathbf{v}$, and $S$ are functions of $t, \mathbf{x}$, whereas the dynamic variables of (1.10) are functions of variables $t, \boldsymbol{\xi}$. But equivalent system (1.7), (1.8), (1.9), and (1.11) of equations for variables $\rho, \mathbf{v}, S$, and $\boldsymbol{\xi}$, which depend on $t, \mathbf{x}$, is the whole system of dynamic equations. This system is obtained from the variational principle, whereas the Euler system (1.7), (1.8), and (1.9) of hydrodynamic equations can be obtained from the variational principle only for the case of irrotational flow. The system (1.7), (1.8), and (1.9) is a closed subsystem of the whole system of dynamic equations (1.7), (1.8), (1.9), and (1.11). On the foundation of closure, the Euler system (1.7), (1.8), and (1.9) is considered conventionally as the complete (full) system of hydrodynamic equations, whereas in reality, the Euler system (1.7), (1.8), and (1.9) is only a curtailed system, that is, only a part of the complete (full) system of hydrodynamic equations formed by (1.7), (1.8), (1.9), and (1.11). If we work with the Euler system only, we cannot integrate it, in general. If, nevertheless, we integrate it in some special cases, the arbitrary functions of Clebsch potentials $\boldsymbol{\xi}$ appear in integrated dynamic equations. If we use the complete system (1.7), (1.8), (1.9), and (1.11), we can always integrate it and reduce the number of dynamic equations. This integration is accompanied by appearance of three arbitrary functions $\mathbf{g}(\boldsymbol{\xi})=\left\{\boldsymbol{g}^{\alpha}(\boldsymbol{\xi})\right\}, \alpha=1,2,3$, of Clebsch potentials $\boldsymbol{\xi}$ in dynamic equations. These functions contain full information on initial and boundary conditions for the fluid flow.

The integrated dynamic equations have different form for the irrotational and rotational flows. In particular, if $\nabla \times \mathbf{g}=0$, what corresponds to irrotational flow of barotropic fluid (1.11), known as Lin constraints [13], are not dynamic equations in the integrated system of dynamic equations, and the variables $\boldsymbol{\xi}$ have no relation to the calculation of the fluid flow. In the general case of rotational flow, when $\nabla \times \mathbf{g} \neq 0$ identically, equations (1.11) are dynamic equations for the fluid. We show this as soon as the corresponding mathematical technique is developed.

In Section 2, Jacobian technique is considered. Section 3 is devoted to derivation of hydrodynamic equations of the ideal fluid from the variational principle. The variational principle for incompressible fluid is considered in Section 4. In Section 5, twodimensional flow of incompressible fluid is described in terms of GSF. In Section 6, the inflow conditions for the stationary two-dimensional flow of incompressible fluid are 
introduced. Section 7 is devoted to stationary flow around an obstacle. Some examples of two-dimensional stationary flow of incompressible fluid are considered in Section 8.

2. Jacobian technique. We consider such a space-time symmetric mathematical object as the Jacobian:

$$
J \equiv \frac{\partial\left(\xi_{0}, \xi_{1}, \xi_{2}, \xi_{3}\right)}{\partial\left(x^{0}, x^{1}, x^{2}, x^{3}\right)} \equiv \operatorname{det}\left\|\xi_{i, k}\right\|, \quad \xi_{i, k} \equiv \partial_{k} \xi_{i} \equiv \frac{\partial \xi_{i}}{\partial x^{k}}, i, k=0,1,2,3
$$

Here $\xi=\left\{\xi_{0}, \boldsymbol{\xi}\right\}=\left\{\xi_{0}, \xi_{1}, \xi_{2}, \xi_{3}\right\}$ are four scalar considered to be functions of $x=$ $\left\{x^{0}, \mathbf{x}\right\}, \xi=\xi(x)$. The functions $\left\{\xi_{0}, \xi_{1}, \xi_{2}, \xi_{3}\right\}$ are supposed to be independent in the sense that $J \neq 0$. It is useful to consider the Jacobian $J$ as 4 -linear function of variables $\xi_{i, k} \equiv \partial_{k} \xi_{i}, i, k=0,1,2,3$. Then one can introduce derivatives of $J$ with respect to $\xi_{i, k}$. The derivative $\partial J / \partial \xi_{i, k}$ appears as a result of a replacement of $\xi_{i}$ by $x^{k}$ in the relation (2.1):

$$
\frac{\partial J}{\partial \xi_{i, k}} \equiv \frac{\partial\left(\xi_{0}, \ldots, \xi_{i-1}, x^{k}, \xi_{i+1}, \ldots, \xi_{3}\right)}{\partial\left(x^{0}, x^{1}, x^{2}, x^{3}\right)}, \quad i, k=0,1,2,3
$$

For instance,

$$
\frac{\partial J}{\partial \xi_{0, i}} \equiv \frac{\partial\left(x^{i}, \xi_{1}, \xi_{2}, \xi_{3}\right)}{\partial\left(x^{0}, x^{1}, x^{2}, x^{3}\right)}, \quad i=0,1,2,3
$$

This rule is valid for higher derivatives of $J$ also:

$$
\begin{aligned}
\frac{\partial^{2} J}{\partial \xi_{i, k} \partial \xi_{s, l}} & \equiv \frac{\partial\left(\xi_{0}, \ldots, \xi_{i-1}, x^{k}, \xi_{i+1}, \ldots, \xi_{s-1}, x^{l}, \xi_{s+1}, \ldots, \xi_{3}\right)}{\partial\left(x^{0}, x^{1}, x^{2}, x^{3}\right)} \\
& \equiv \frac{\partial\left(x^{k}, x^{l}\right)}{\partial\left(\xi_{i}, \xi_{s}\right)} \frac{\partial\left(\xi_{0}, \xi_{1}, \xi_{2}, \xi_{3}\right)}{\partial\left(x^{0}, x^{1}, x^{2}, x^{3}\right)} \equiv J\left(\frac{\partial x^{k}}{\partial \xi_{i}} \frac{\partial x^{l}}{\partial \xi_{s}}-\frac{\partial x^{k}}{\partial \xi_{s}} \frac{\partial x^{l}}{\partial \xi_{i}}\right), \quad i, k, l, s=0,1,2,3 .
\end{aligned}
$$

It follows from (2.1) and (2.2) that

$$
\begin{aligned}
\frac{\partial x^{k}}{\partial \xi_{i}} & \equiv \frac{\partial\left(\xi_{0}, \ldots, \xi_{i-1}, x^{k}, \xi_{i+1}, \ldots, \xi_{3}\right)}{\partial\left(\xi_{0}, \xi_{1}, \xi_{2}, \xi_{3}\right)} \\
& \equiv \frac{\partial\left(\xi_{0}, \ldots, \xi_{i-1}, x^{k}, \xi_{i+1}, \ldots, \xi_{3}\right)}{\partial\left(x^{0}, x^{1}, x^{2}, x^{3}\right)} \frac{\partial\left(x^{0}, x^{1}, x^{2}, x^{3}\right)}{\partial\left(\xi_{0}, \xi_{1}, \xi_{2}, \xi_{3}\right)} \\
& \equiv \frac{1}{J} \frac{\partial J}{\partial \xi_{i, k}}, \quad i, k=0,1,2,3
\end{aligned}
$$

and (2.4) may be written in the form

$$
\frac{\partial^{2} J}{\partial \xi_{i, k} \partial \xi_{s, l}} \equiv \frac{1}{J}\left(\frac{\partial J}{\partial \xi_{i, k}} \frac{\partial J}{\partial \xi_{s, l}}-\frac{\partial J}{\partial \xi_{i, l}} \frac{\partial J}{\partial \xi_{s, k}}\right), \quad i, k, l, s=0,1,2,3 .
$$


The derivative $\partial J / \partial \xi_{i, k}$ is a cofactor to the element $\xi_{i, k}$ of the determinant (2.1). Then one has the following identities:

$$
\begin{array}{r}
\xi_{l, k} \frac{\partial J}{\partial \xi_{s, k}} \equiv \delta_{l}^{s} J, \quad \xi_{k, l} \frac{\partial J}{\partial \xi_{k, s}} \equiv \delta_{l}^{s} J, \quad l, s=0,1,2,3, \\
\partial_{k} \frac{\partial J}{\partial \xi_{i, k}} \equiv \frac{\partial^{2} J}{\partial \xi_{i, k} \partial \xi_{s, l}} \partial_{k} \partial_{l} \xi_{s} \equiv 0, \quad i=0,1,2,3 .
\end{array}
$$

Here and in what follows, a summation over two repeated indices is produced $(0-3)$ for Latin indices. The identity (2.8) can be considered as a corollary of the identity (2.6) and a symmetry of $\partial_{k} \partial_{l} \xi_{s}$ with respect to permutation of indices $k$, $l$. Convolution of (2.6) with $\partial_{k}$ or $\partial_{l}$ vanishes also:

$$
\partial_{k} \frac{\partial^{2} J}{\partial \xi_{i, k} \partial \xi_{s, l}} \equiv \frac{\partial^{3} J}{\partial \xi_{i, k} \partial \xi_{s, l} \partial \xi_{m, n}} \partial_{k} \partial_{n} \xi_{m} \equiv 0, \quad i, l, s=0,1,2,3 .
$$

Relations (2.1), (2.2), (2.3), (2.4), (2.5), and (2.6) are written for four independent variables $x$, but they are valid in an evident way for arbitrary number of $n+1$ variables $x=\left\{x^{0}, x^{1}, \ldots, x^{n}\right\}$ and $\xi=\left\{\xi_{0}, \boldsymbol{\xi}\right\}, \boldsymbol{\xi}=\left\{\xi_{1}, \xi_{2}, \ldots, \xi_{n}\right\}$.

Application of the Jacobian $J$ to hydrodynamics is founded on the property, which can be formulated as the property of the GSF $\boldsymbol{\xi}=\left\{\xi_{1}, \xi_{2}, \ldots, \xi_{n}\right\}$ in the $(n+1)$-dimensional space of coordinates $x=\left\{x^{0}, x^{1}, \ldots, x^{n}\right\}$.

On the basis of the GSF $\boldsymbol{\xi}$, one can construct $n$-vector $j^{i}$ :

$$
j^{i}=m \frac{\partial J}{\partial \xi_{0, i}}, \quad j^{i}=\{\rho, \rho \mathbf{v}\}, m=\text { const }
$$

in such a way that $j^{i}$ satisfies the continuity equation

$$
\partial_{i} j^{i}=0
$$

identically for any choice of variables $\boldsymbol{\xi}$. Besides, the variables $\boldsymbol{\xi}$ are constant along any line $\mathscr{L}$ tangent to $n$-vector $j^{i}$, and can label this line because the set of quantities $\xi$ is different for different lines $\mathscr{L}$.

In the case of dynamical system (1.7), (1.8), (1.9), and (1.11), we have $j^{i}=\{\rho, \rho \mathbf{v}\}$. It means that (1.7), (1.11) are satisfied at any choice of the GSF (Clebsch potentials) $\boldsymbol{\xi}$. Substituting the flux vector $j^{i}=\{\rho, \rho \mathbf{v}\}$, expressed via $\boldsymbol{\xi}$, in the remaining hydrodynamic equations, we obtain dynamic equations for determination of the GSF $\boldsymbol{\xi}$. This procedure is insignificant in the case of irrotational flow, when Lin constraints (1.11) are of no importance. But it is a very effective procedure in the case of rotational flow because it reduces the number of dynamic equations to be solved.

The continuity equation (2.11) is used without approximation in all hydrodynamic models, and the change of variables $\{\rho, \rho \mathbf{v}\} \leftrightarrow \boldsymbol{\xi}$ described by (2.10) appears sometimes to be useful. In particular, in the case of two-dimensional established flow of incompressible fluid, the GSF $\boldsymbol{\xi}$ reduces to one variable $\xi_{2}=\psi$, known as the stream function. In this case, there are only two essential independent variables $x^{0}=x$ and $x^{1}=y$, and 
the relations (2.10) and (2.11) reduce to relations

$$
\rho^{-1} j_{x}=u=-\frac{\partial \psi}{\partial y}, \quad \rho^{-1} j_{y}=v=\frac{\partial \psi}{\partial x}, \quad \frac{\partial u}{\partial x}+\frac{\partial v}{\partial y}=0
$$

Defining the stream line as a line tangent to the flux $j$ :

$$
\frac{d x}{j_{x}}=\frac{d y}{j_{y}}
$$

we deduce that the stream function is constant along the stream line because according to two first equations of (2.12), $\psi=\psi(x, y)$ is an integral of (2.13).

In the general case, when the space dimension is $n, x=\left\{x^{0}, x^{1}, \ldots, x^{n}\right\}$, and $\xi=$ $\left\{\xi_{0}, \boldsymbol{\xi}\right\}, \boldsymbol{\xi}=\left\{\xi_{1}, \xi_{2}, \ldots, \xi_{n}\right\}$, the quantities $\boldsymbol{\xi}=\left\{\xi_{\alpha}\right\}, \alpha=1,2, \ldots, n$, are constant along the line $\mathscr{L}: x=x(\tau)$ tangent to the flux vector $j=\left\{j^{i}\right\}, i=0,1, \ldots, n$ :

$$
\mathscr{L}: \frac{d x^{i}}{d \tau}=j^{i}(x), \quad i=0,1, \ldots, n,
$$

where $\tau$ is a parameter along the line $\mathscr{L}$. This statement is formulated mathematically in the form

$$
\frac{d \xi_{\alpha}}{d \tau}=j^{i} \partial_{i} \xi_{\alpha}=m \frac{\partial J}{\partial \xi_{0, i}} \partial_{i} \xi_{\alpha}=0, \quad \alpha=1,2, \ldots, n
$$

The last equality follows from the first identity (2.7) taken for $s=0$, and $l=1,2, \ldots, n$. Interpretation of the line (2.14) tangent to the flux is different for different cases. If $x=\left\{x^{0}, x^{1}, \ldots, x^{n}\right\}$ contains only spatial coordinates, the line (2.14) is a line in the usual space. It is regarded as a stream line, and $\boldsymbol{\xi}$ can be interpreted as quantities which are constant along the stream line (i.e., as a GSF). If $x^{0}$ is the time coordinate, (2.14) describes a line in the space-time. This line (known as a world line of a fluid particle) determines a motion of the fluid particle. Variables $\boldsymbol{\xi}=\left\{\boldsymbol{\xi}_{1}, \boldsymbol{\xi}_{2}, \ldots, \boldsymbol{\xi}_{n}\right\}$ which are constant along the world line are different, generally, for different particles. If $\xi_{\alpha}, \alpha=$ $1,2, \ldots, n$, are independent, they may be used for the fluid particle labeling. When one of coordinates $x$ is time-like, the set of variables $\xi$ is not perceived as a generalization of the stream function $\psi$. Nevertheless, we will use the term GSF in all cases because from mathematical viewpoint, it is of no importance whether coordinate $x^{0}$ is time-like or space-like.

Thus, although interpretation of the relation (2.10) considered as a change of dependent variables $j$ by $\boldsymbol{\xi}$ may be different, from the mathematical viewpoint, this transformation means a replacement of the continuity equation by some equations for the GSF $\boldsymbol{\xi}$. Difference of the interpretation is of no importance in this context.

Note that the expressions

$$
j^{i}=m \rho_{0}(\boldsymbol{\xi}) \frac{\partial J}{\partial \xi_{0, i}} \equiv m \rho_{0}(\boldsymbol{\xi}) \frac{\partial\left(x^{i}, \xi_{1}, \xi_{2}, \xi_{3}\right)}{\partial\left(x^{0}, x^{1}, x^{2}, x^{3}\right)}, \quad i=0,1,2,3
$$


can be also considered as four-flux satisfying the continuity equation (2.11). Here $m$ is a constant and $\rho_{0}(\boldsymbol{\xi})$ is an arbitrary function of $\boldsymbol{\xi}$. It follows from the identity

$$
m \rho_{0}(\boldsymbol{\xi}) \frac{\partial\left(x^{i}, \xi_{1}, \xi_{2}, \xi_{3}\right)}{\partial\left(x^{0}, x^{1}, x^{2}, x^{3}\right)} \equiv m \frac{\partial\left(x^{i}, \tilde{\xi}_{1}, \xi_{2}, \xi_{3}\right)}{\partial\left(x^{0}, x^{1}, x^{2}, x^{3}\right)}, \quad \tilde{\xi}_{1}=\int_{0}^{\xi_{1}} \rho_{0}\left(\xi_{1}^{\prime}, \xi_{2}, \xi_{3}\right) d \xi_{1}^{\prime}
$$

The Jacobian technique is very useful for manipulation with hydrodynamic equations (1.7), (1.8), (1.9), and (1.11). For instance, one can integrate the complete system of $5+3$ dynamic equations (1.7), (1.8), (1.9), and (1.11), reducing it to the system of five dynamic equations written in the form [15]

$$
\begin{gathered}
S(t, \mathbf{x})=S_{0}(\boldsymbol{\xi}), \\
\rho(t, \mathbf{x})=\rho_{0}(\boldsymbol{\xi}) \frac{\partial\left(\xi_{1}, \xi_{2}, \xi_{3}\right)}{\partial\left(x^{1}, x^{2}, x^{3}\right)} \equiv \rho_{0}(\boldsymbol{\xi}) \frac{\partial(\boldsymbol{\xi})}{\partial(\mathbf{x})}, \\
\mathbf{v}(t, \mathbf{x})=\mathbf{u}(\varphi, \boldsymbol{\xi}, \eta, S) \equiv \nabla \varphi+g^{\alpha}(\boldsymbol{\xi}) \nabla \xi_{\alpha}-\eta \nabla S,
\end{gathered}
$$

where $S_{0}(\boldsymbol{\xi}), \rho_{0}(\boldsymbol{\xi})$, and $\boldsymbol{g}(\boldsymbol{\xi})=\left\{g^{\alpha}(\boldsymbol{\xi})\right\}, \alpha=1,2,3$, are arbitrary integration functions of argument $\xi$. The quantities $\varphi, \eta$ are new dependent variables satisfying dynamic equations

$$
\begin{gathered}
\frac{\partial \varphi}{\partial t}+\mathbf{u}(\varphi, \boldsymbol{\xi}, \eta, S) \nabla \varphi-\frac{1}{2}[\mathbf{u}(\varphi, \boldsymbol{\xi}, \eta, S)]^{2}+\frac{\partial(\rho E)}{\partial \rho}=0, \\
\frac{\partial \eta}{\partial t}+\mathbf{u}(\varphi, \boldsymbol{\xi}, \eta, S) \nabla \eta=-\frac{\partial E}{\partial S} .
\end{gathered}
$$

If five dependent variables $\varphi, \boldsymbol{\xi}$, and $\eta$ satisfy the system of equations (1.11), (2.21), and (2.22), five dynamic variables $S, \rho, \mathbf{v}$ in (2.18), (2.19), and (2.20) satisfy dynamic equations (1.7), (1.8), and (1.9). Indefinite functions $S_{0}(\boldsymbol{\xi}), \rho_{0}(\boldsymbol{\xi})$, and $\boldsymbol{g}^{\alpha}(\boldsymbol{\xi})$ can be determined from initial and boundary conditions in a way such that the initial and boundary conditions for variables $\varphi, \xi$, and $\eta$ were universal in the sense that they do not depend on the fluid flow [15]. Further dynamic equations (1.11), (2.21), and (2.22) will be derived directly from the variational principle.

3. Variational principle. The ideal (nondissipative) fluid is a continuous dynamical system whose dynamic equations can be derived from the variational principle with the action functional

$$
\mathscr{A}_{\mathrm{L}}[\mathbf{x}]=\int\left\{\frac{m}{2}\left(\frac{d \mathbf{x}}{d t}\right)^{2}-V\right\} \rho_{0}(\boldsymbol{\xi}) d t d \boldsymbol{\xi}
$$

where the fluid particle coordinates $\mathbf{x}=\left\{x^{\alpha}(t, \boldsymbol{\xi})\right\}, \alpha=1,2,3$, are dependent variables considered to be functions of time $t$ and of labels (Lagrangian coordinates) $\boldsymbol{\xi}=$ $\left\{\xi_{1}, \xi_{2}, \xi_{3}\right\}, d \mathbf{x} / d t$ is a derivative of $\mathbf{x}$ with respect to $t$ taken at fixed $\boldsymbol{\xi}$ :

$$
\frac{d x^{\alpha}}{d t} \equiv \frac{\partial\left(x^{\alpha}, \xi_{1}, \xi_{2}, \xi_{3}\right)}{\partial\left(t, \xi_{1}, \xi_{2}, \xi_{3}\right)} \equiv \frac{\partial\left(x^{\alpha}, \boldsymbol{\xi}\right)}{\partial(t, \boldsymbol{\xi})}, \quad \alpha=1,2,3,
$$


$\rho_{0}(\boldsymbol{\xi})$ is some nonnegative weight function, and $V$ is a potential of a self-consistent force field which depends on $\boldsymbol{\xi}, \mathbf{x}$, and derivatives of $\mathbf{x}$ with respect to $\boldsymbol{\xi}$. A mass of the fluid particle $m=$ const. The value of mass is unessential, and without loss of generality, one may set $m=1$. For the ideal fluid, the potential $V$ is such a function of $\boldsymbol{\xi}$ and $\partial \mathbf{x} / \partial \boldsymbol{\xi}$ which can be represented in the form

$$
V\left(\boldsymbol{\xi}, \frac{\partial \mathbf{x}}{\partial \boldsymbol{\xi}}\right)=m E(\rho, S), \quad \rho=\rho_{0}(\boldsymbol{\xi})\left(\frac{\partial\left(x^{1}, x^{2}, x^{3}\right)}{\partial\left(\xi_{1}, \xi_{2}, \xi_{3}\right)}\right)^{-1}, S=S_{0}(\boldsymbol{\xi}),
$$

where the entropy per unit mass $S=S_{0}(\boldsymbol{\xi})$ is a given function of $\boldsymbol{\xi}$ determined by the initial conditions, $\rho$ is the fluid density, and $E(\rho, S)$ is the internal energy of the fluid per unit mass of the fluid. The quantity $E(\rho, S)$ is considered to be a given function of its arguments.

The variational principle (3.1) generates the sixth-order system of dynamic equations for six dependent variables $\mathbf{x}, d \mathbf{x} / d t$, considered to be functions of independent variables $t, \boldsymbol{\xi}$. Such a way of description is known as the Lagrangian description of the fluid. If the variables $t, \mathbf{x}$ are considered to be independent variables, and six variables $\xi, \partial \xi / \partial t$, are considered to be dependent variables, the sixth-order system of dynamic equations for $\xi, \partial \xi / \partial t$, arises. It is known as the Eulerian description of the fluid.

The partial choice of labeling the fluid particles by variables $\boldsymbol{\xi}$ is unessential from a physical point of view. This circumstance is displayed in an existence of the relabeling group

$$
\xi_{\alpha} \longrightarrow \tilde{\xi}_{\alpha}=\tilde{\xi}_{\alpha}(\boldsymbol{\xi}), \quad D \equiv \operatorname{det}\left\|\frac{\partial \tilde{\xi}_{\alpha}}{\partial \xi_{\beta}}\right\| \neq 0, \quad \alpha, \beta=1,2,3
$$

The action (3.1) appears to be invariant with respect to the relabeling group, provided that $V$ has the form (3.3), where $S$ is a scalar and $\rho(\xi)$ is a scalar density, that is, under the transformations (3.4), $\rho_{0}(\boldsymbol{\xi})$ transforms as follows:

$$
\rho_{0}(\boldsymbol{\xi}) \longrightarrow \tilde{\rho}_{0}(\tilde{\boldsymbol{\xi}}), \quad \rho(\boldsymbol{\xi}) \longrightarrow \tilde{\rho}(\tilde{\boldsymbol{\xi}})=\rho(\boldsymbol{\xi}) D
$$

The relabeling group is used in hydrodynamics comparatively recently $[2,3,7,8,10$, $17,20]$.

The relabeling group is a symmetry group of the dynamical system. It may be used to simplify a description of the fluid. There are at least two different ways of usage of the relabeling group. The first (conventional) way of such a simplification is a use of five relabeling-invariant variables $\mathbf{v}=d \mathbf{x} / d t, \rho$, and $S$, considered to be functions of independent variables $t, \mathbf{x}$. This way of description will be referred to as the relabeling invariant description (RID). The variables $\boldsymbol{\xi}$, describing labeling, are eliminated. At this elimination of $\boldsymbol{\xi}$, the dynamic equation (1.11) is eliminated also on the foundation that the remaining Eulerian system of dynamic equations (1.7), (1.8), and (1.9) for five dependent dynamic variables $\mathbf{v}, \rho$, and $S$ is closed. Conventionally, most researchers use RID, 
and hence the Eulerian system of dynamic equations. Another way of simplification is a use of the relabeling group for integration of the complete system of dynamic equations (1.7), (1.8), (1.9), and (1.11) and application of the system (1.11), (2.21), and (2.22).

In general, equivalency of the system (1.11), (2.21), and (2.22) and the system (1.7), (1.8), (1.9), and (1.11) can be verified by a direct substitution of variables $\rho, S$, and $\mathbf{v}$, defined by the relations (2.18), (2.19), and (2.20), into equations (1.7), (1.8), and (1.9). Using (1.11), (2.21), and (2.22), one obtains identities after subsequent calculations. But such computations do not display a connection between the integration and the invariance with respect to the relabeling group (3.4). Besides, a meaning of new variables $\varphi, \eta$ is not clear.

We will use for our investigations the variational principle (3.1). Note that for a long time, a derivation of a variational principle for hydrodynamic equations (1.7), (1.8), and (1.9) existed as an independent problem [2, 6, 8, 11, 13, 18, 19]. Existence of this problem was connected with a lack of understanding that the system of hydrodynamic equations (1.7), (1.8), and (1.9) is a curtailed system, and the full system of dynamic equations (1.7), (1.8), (1.9), and (1.11) includes (1.11) that describes a motion of the fluid particles in the given velocity field. The variational principle can generate only the complete system of dynamic variables (but not its closed subsystem). Without understanding, this one tried to form the Lagrangian for the system (1.7), (1.8), and (1.9) as a sum of some quantities taken with Lagrange multipliers. The left-hand side of dynamic equations (1.7), (1.8), and (1.9) and some other constraints were taken as such quantities.

Now this problem has been solved (see review by Salmon [18]) on the basis of the Eulerian version of the variational principle (3.1), where (1.11) appear automatically and cannot be ignored. In our version of the variational principle, we follow Salmon [18] with some modifications which underline a curtailed character of hydrodynamic equations (1.7), (1.8), and (1.9) because the understanding of the curtailed character of the system (1.7), (1.8), and (1.9) removes the problem of derivation of the variational principle for the hydrodynamic equations (1.7), (1.8), and (1.9).

The starting point is the action (3.1). We prefer to work with Eulerian description when Lagrangian coordinates (particle labeling) $\boldsymbol{\xi}=\left\{\boldsymbol{\xi}_{0}, \boldsymbol{\xi}\right\}, \boldsymbol{\xi}=\left\{\boldsymbol{\xi}_{1}, \boldsymbol{\xi}_{2}, \boldsymbol{\xi}_{3}\right\}$, are considered to be dependent variables and Eulerian coordinates $x=\left\{x^{0}, \mathbf{x}\right\}=\{t, \mathbf{x}\}, \mathbf{x}=$ $\left\{x^{1}, x^{2}, x^{3}\right\}$, are considered to be independent variables. Here $\xi_{0}$ is a temporal Lagrangian coordinate which evolves along the particle trajectory in an arbitrary way. Now $\xi_{0}$ is a fictitious variable, but after integration of equations, the variable $\xi_{0}$ ceases to be fictitious and turns into the variable $\varphi$ appearing in the integrated system (1.11), (2.21), and (2.22).

Further, mainly space-time symmetric designations will be used that simplifies considerably all computations. In the Eulerian description, the action functional (3.1) is to be represented as an integral over independent variables $x=\left\{x^{0}, \mathbf{x}\right\}=\{t, \mathbf{x}\}$. We use the Jacobian technique for such a transformation of the action (3.1).

We note that according to (2.5), the derivative (3.2) can be written in the form

$$
v^{\alpha}=\frac{d x^{\alpha}}{d t} \equiv \frac{\partial J}{\partial \xi_{0, \alpha}}\left(\frac{\partial J}{\partial \xi_{0,0}}\right)^{-1}, \quad \alpha=1,2,3
$$


Then components of the four-flux $j=\left\{j^{0}, \mathbf{j}\right\} \equiv\{\rho, \rho \mathbf{v}\}$ can be written in the form (2.16), provided that the designation (2.19):

$$
j^{0}=\rho=m \rho_{0}(\boldsymbol{\xi}) \frac{\partial J}{\partial \xi_{0,0}} \equiv m \rho_{0}(\boldsymbol{\xi}) \frac{\partial\left(x^{0}, \xi_{1}, \xi_{2}, \xi_{3}\right)}{\partial\left(x^{0}, x^{1}, x^{2}, x^{3}\right)}
$$

is used.

At such a form of the mass density $\rho$, the four-flux $j=\left\{j^{i}\right\}, i=0,1,2,3$, satisfies identically the continuity equation (2.11) which takes place in virtue of identities (2.7), (2.8). Besides, in virtue of identities (2.7), (2.8), the Lin constraints (1.11) are fulfilled identically:

$$
j^{i} \partial_{i} \xi_{\alpha}=m \rho_{0}(\boldsymbol{\xi}) \frac{\partial J}{\partial \xi_{0, i}} \partial_{i} \xi_{\alpha} \equiv 0, \quad \alpha=1,2,3
$$

Components $j^{i}$ are invariant with respect to the relabeling group (3.4), provided that the function $\rho_{0}(\boldsymbol{\xi})$ transforms according to (3.5).

One has

$$
\begin{aligned}
\rho_{0}(\boldsymbol{\xi}) d t d \boldsymbol{\xi} & =\rho_{0}(\boldsymbol{\xi}) \frac{\partial J}{\partial \xi_{0,0}} d t d \mathbf{x}=\frac{\rho}{m} d t d \mathbf{x}, \\
\frac{m}{2}\left(\frac{d x^{\alpha}}{d t}\right)^{2} & =\frac{m}{2}\left(\frac{\partial J}{\partial \xi_{0, \alpha}}\right)^{2}\left(\frac{\partial J}{\partial \xi_{0,0}}\right)^{-2}
\end{aligned}
$$

and the variational problem with the action functional (3.1) is written as a variational problem with the action functional

$$
\mathscr{A}_{\mathrm{E}}[\boldsymbol{\xi}]=\int\left\{\frac{1}{2}\left(\frac{\partial J}{\partial \xi_{0, \alpha}}\right)^{2}\left(\frac{\partial J}{\partial \xi_{0,0}}\right)^{-2}-E\right\} \rho d t d \mathbf{x},
$$

where $\rho$ is a fixed function of $\xi=\left\{\xi_{0}, \xi\right\}$ and of $\xi_{\alpha, i} \equiv \partial_{i} \xi_{\alpha}, \alpha=1,2,3, i=0,1,2,3$, defined by (3.7), and $E$ is the internal energy of the fluid which is supposed to be a fixed function of $\rho$ and $S_{0}(\boldsymbol{\xi})$ :

$$
E=E\left(\rho, S_{0}(\boldsymbol{\xi})\right)
$$

where $\rho$ is defined by (3.7) and $S_{0}(\boldsymbol{\xi})$ is some fixed function of $\boldsymbol{\xi}$, describing initial distribution of the entropy over the fluid.

The action (3.10) is invariant with respect to subgroup $\mathscr{G}_{S_{0}}$ of the relabeling group (3.4). The subgroup $\mathscr{G}_{S_{0}}$ is determined in such a way that any surface $S_{0}(\boldsymbol{\xi})=$ const is invariant with respect to $\mathscr{G}_{S_{0}}$. In general, the subgroup $\mathscr{G}_{S_{0}}$ is determined by two arbitrary functions of $\boldsymbol{\xi}$.

The action (3.10) generates the sixth-order system of dynamic equations, consisting of three second-order equations for three dependent variables $\boldsymbol{\xi}$. Invariance of the action (3.10) with respect to the subgroup $\mathscr{G}_{S_{0}}$ allows one to integrate the system of dynamic equations. The order of the system becomes reduced, and two arbitrary integration functions appear. The order of the system reduces to five (but not to four) 
because the fictitious dependent variable $\xi_{0}$ ceases to be fictitious as a result of the integration.

Unfortunately, the subgroup $\mathscr{G}_{S_{0}}$ depends on the form of the function $S_{0}(\boldsymbol{\xi})$ and cannot be obtained in a general form. In the special case when $S_{0}(\boldsymbol{\xi})$ does not depend on $\boldsymbol{\xi}$, the subgroup $\mathscr{G}_{S_{0}}$ coincides with the whole relabeling group $\mathscr{G}$, and the order of the integrated system reduces to four.

In the general case, it is convenient to introduce a new dependent variable

$$
S=S_{0}(\boldsymbol{\xi})
$$

Addition of the new variable increases the number of dynamic variables, but at the same time, this addition makes the action to be invariant with respect to the whole relabeling group $\mathscr{G}$. It allows one to integrate the dynamic system and to reduce the number of dynamic variables. According to (3.8), the variable $S$ satisfies the dynamic equation (1.9):

$$
j^{i} \partial_{i} S=0
$$

In virtue of designations (2.16) and identities (2.7), (2.8), equations (3.8) and (3.13) are fulfilled identically. Hence, they can be added to the action functional (3.10) as side constraints without a change of the variational problem. Adding (3.13) to the Lagrangian of the action (3.10) by means of a Lagrange multiplier $\eta$, one obtains

$$
\mathscr{A}_{\mathrm{E}}[\boldsymbol{\xi}, \eta, S]=\int\left\{\frac{\rho}{2}\left(\frac{\partial J}{\partial \xi_{0, \alpha}}\right)^{2}\left(\frac{\partial J}{\partial \xi_{0,0}}\right)^{-1}-\rho E+\eta \frac{\partial J}{\partial \xi_{0, k}} \partial_{k} S\right\} d t d \mathbf{x}
$$

where $E=E(\rho, S)$. The action (3.14) is invariant with respect to the relabeling group $\mathscr{G}$ which is determined by three arbitrary functions of $\boldsymbol{\xi}$.

To obtain the dynamic equations, it is convenient to introduce new dependent variables $j^{i}$, defined by (2.16). We introduce the new variables $j^{i}$ by means of designations (2.16) taken with the Lagrange multipliers $p_{i}, i=0,1,2,3$. Consideration of (2.16) as side constraints does not change the variational problem because conditions (2.16) are always compatible with dynamic equations generated by the action (3.14). Then the action (3.14) takes the form

$$
\mathscr{A}_{\mathrm{E}}[\rho, \mathbf{j}, \boldsymbol{\xi}, p, \eta, S]=\int\left\{\frac{\mathbf{j}^{2}}{2 \rho}-\rho E-p_{k}\left(j^{k}-m \rho_{0}(\boldsymbol{\xi}) \frac{\partial J}{\partial \xi_{0, k}}\right)+\eta j^{k} \partial_{k} S\right\} d t d \mathbf{x}
$$

For obtaining dynamic equations, the variables $\rho, \mathbf{j}, \boldsymbol{\xi}, p, \eta$, and $S$ are to be varied. We eliminate the variables $p_{i}$ from the action (3.15). Dynamic equations arising as a result of a variation with respect to $\xi_{\alpha}$ have the form

$$
\frac{\delta \mathscr{A}_{\mathrm{E}}}{\delta \xi_{\alpha}} \equiv \hat{\mathscr{L}}_{\alpha} p=-m \partial_{k}\left(\rho_{0}(\boldsymbol{\xi}) \frac{\partial^{2} J}{\partial \xi_{0, i} \partial \xi_{\alpha, k}} p_{i}\right)+m \frac{\partial \rho_{0}(\boldsymbol{\xi})}{\partial \xi_{\alpha}} \frac{\partial J}{\partial \xi_{0, k}} p_{k}=0, \quad \alpha=1,2,3,
$$


where $\hat{\mathscr{L}}_{\alpha}$ are linear operators acting on variables $p=\left\{p_{i}\right\}, i=0,1,2,3$. These equations can be integrated in the form

$$
p_{i}=g^{0}\left(\xi_{0}\right) \partial_{i} \xi_{0}+g^{\alpha}(\xi) \partial_{i} \xi_{\alpha}, \quad i=0,1,2,3
$$

where $\xi_{0}$ is some new variable (temporal Lagrangian coordinate), $g^{\alpha}(\boldsymbol{\xi}), \alpha=1,2,3$, are arbitrary functions of the label $\boldsymbol{\xi}$, and $g^{0}\left(\xi_{0}\right)$ is an arbitrary function of $\xi_{0}$. The relations (3.17) satisfy (3.16) identically. Indeed, substituting (3.17) into (3.16) and using identities (2.6) and (2.7), we obtain

$$
-m \partial_{k}\left\{\rho_{0}(\boldsymbol{\xi})\left[\frac{\partial J}{\partial \xi_{\alpha, k}} g^{0}\left(\xi_{0}\right)-\frac{\partial J}{\partial \xi_{0, k}} g^{\alpha}(\boldsymbol{\xi})\right]\right\}+m \frac{\partial \rho_{0}(\boldsymbol{\xi})}{\partial \xi_{\alpha}} J g^{0}\left(\xi_{0}\right)=0, \quad \alpha=1,2,3
$$

Differentiating braces and using identities (2.7), (2.8), one concludes that (3.18) is an identity.

Setting for simplicity

$$
\partial_{k} \varphi=g^{0}\left(\xi_{0}\right) \partial_{k} \xi_{0}, \quad k=0,1,2,3
$$

we obtain

$$
p_{k}=\partial_{k} \varphi+g^{\alpha}(\xi) \partial_{k} \xi_{\alpha}, \quad k=0,1,2,3
$$

Note that integration of (3.16) by means of the Jacobian technique and appearance of arbitrary functions $g^{\alpha}(\boldsymbol{\xi})$ is a result of invariance of the action with respect to the relabeling group (3.4).

Substituting (3.20) in (3.15), one can eliminate variables $p_{i}, i=0,1,2,3$, from the functional (3.15). The term $\boldsymbol{g}^{\alpha}(\boldsymbol{\xi}) \partial_{k} \xi_{\alpha} \partial J / \partial \xi_{0, k}$ vanishes, the term $\partial_{k} \varphi \partial J / \partial \xi_{0, k}$ makes no contribution to the dynamic equations. The action functional takes the form

$$
\mathscr{A}_{\mathrm{g}}[\rho, \mathbf{j}, \boldsymbol{\xi}, \eta, S]=\int\left\{\frac{\mathbf{j}^{2}}{2 \rho}-\rho E-j^{k}\left(\partial_{k} \varphi+g^{\alpha}(\boldsymbol{\xi}) \partial_{k} \xi_{\alpha}-\eta \partial_{k} S\right)\right\} d t d \mathbf{x}
$$

where $g^{\alpha}(\boldsymbol{\xi})$ are considered to be fixed functions of $\boldsymbol{\xi}$ which are determined from initial conditions. Varying the action (3.21) with respect to $\varphi, \boldsymbol{\xi}, \eta, S$, j, and $\rho$, we obtain dynamic equations

$$
\begin{gathered}
\delta \varphi: \partial_{k} j^{k}=0, \\
\delta \xi_{\alpha}: \Omega^{\alpha \beta} j^{k} \partial_{k} \xi_{\beta}=0, \quad \alpha=1,2,3,
\end{gathered}
$$


where $\Omega^{\alpha \beta}$ is defined by the relations

$$
\begin{gathered}
\Omega^{\alpha \beta}=\frac{\partial g^{\alpha}(\boldsymbol{\xi})}{\partial \xi_{\beta}}-\frac{\partial g^{\beta}(\boldsymbol{\xi})}{\partial \xi_{\alpha}}, \quad \alpha, \beta=1,2,3, \\
\delta \eta: j^{k} \partial_{k} S=0, \\
\delta S: j^{k} \partial_{k} \eta=-\rho \frac{\partial E}{\partial S}, \\
\delta \mathbf{j}: \mathbf{v} \equiv \frac{\mathbf{j}}{\rho}=\nabla \varphi+g^{\alpha}(\boldsymbol{\xi}) \nabla \xi_{\alpha}-\eta \nabla S, \\
\delta \rho:-\frac{\mathbf{j}^{2}}{2 \rho^{2}}-\frac{\partial(\rho E)}{\partial \rho}-\partial_{0} \varphi-g^{\alpha}(\boldsymbol{\xi}) \partial_{0} \xi_{\alpha}+\eta \partial_{0} S=0 .
\end{gathered}
$$

Deriving relations (3.23) and (3.26), the continuity equation (3.22) is used. It is easy to see that (3.32) is equivalent to the Lin constraints (1.11), provided that

$$
\operatorname{det}\left\|\Omega^{\alpha \beta}\right\| \neq 0
$$

If condition (3.29) is obtained and (1.11) are satisfied, (3.22) and (3.25) can be integrated in the form of (2.18) and (2.19), respectively. Equations (3.26) and (3.27) are equivalent to (2.20) and (2.22). Finally, eliminating $\partial_{0} \xi_{\alpha}$ and $\partial_{0} S$ from (3.28) by means of (1.11) and (3.25), we obtain (2.21) and, hence, the system of dynamic equations (1.11), (2.21), and (2.22), where designations (2.18), (2.19), and (2.20) are used.

The curtailed system (1.7), (1.8), and (1.9) can be obtained from (3.22), (3.23), (3.24), (3.25), (3.26), (3.27), and (3.28) as follows. Equations (3.22), (3.25) coincide with (1.7), (1.9). For deriving (1.8), we note that the vorticity $\boldsymbol{\omega} \equiv \nabla \times \mathbf{v}$ and $\mathbf{v} \times \boldsymbol{\omega}$ are obtained from (3.27) in the form

$$
\begin{gathered}
\boldsymbol{\omega}=\nabla \times \mathbf{v}=\frac{1}{2} \Omega^{\alpha \beta} \nabla \xi_{\beta} \times \nabla \xi_{\alpha}-\nabla \eta \times \nabla S, \\
\mathbf{v} \times \boldsymbol{\omega}=\Omega^{\alpha \beta} \nabla \xi_{\beta}(\mathbf{v} \nabla) \xi_{\alpha}+\nabla S(\mathbf{v} \nabla) \eta-\nabla \eta(\mathbf{v} \nabla) S .
\end{gathered}
$$

We form a difference between the time derivative of (3.27) and the gradient of (3.28). Eliminating $\Omega^{\alpha \beta} \partial_{0} \boldsymbol{\xi}_{\alpha}, \partial_{0} S$, and $\partial_{0} \eta$ by means of (3.23), (3.25), and (3.26), one obtains

$$
\begin{aligned}
\partial_{0} \mathbf{v}+\nabla \frac{\mathbf{v}^{2}}{2} & +\frac{\partial^{2}(\rho E)}{\partial \rho^{2}} \nabla \rho+\frac{\partial^{2}(\rho E)}{\partial \rho \partial S} \nabla S-\rho \frac{\partial E}{\partial S} \nabla S \\
& -\Omega^{\alpha \beta} \nabla \xi_{\beta}(\mathbf{v} \nabla) \xi_{\alpha}+\nabla \eta(\mathbf{v} \nabla) S-\nabla S(\mathbf{v} \nabla) \eta=0 .
\end{aligned}
$$

Using (3.30), the expression (3.31) reduces to

$$
\partial_{0} \mathbf{v}+\nabla \frac{\mathbf{v}^{2}}{2}+\frac{1}{\rho} \nabla\left(\rho^{2} \frac{\partial E}{\partial \rho}\right)-\mathbf{v} \times(\nabla \times \mathbf{v})=0
$$

In virtue of the identity

$$
\mathbf{v} \times(\nabla \times \mathbf{v}) \equiv \nabla \frac{\mathbf{v}^{2}}{2}-(\mathbf{v} \nabla) \mathbf{v}
$$

the last equation is equivalent to (1.8). Note that at derivation of the curtailed system (1.7), (1.8), and (1.9), condition (3.29) is not used, and the system (1.7), (1.8), and (1.9) is valid in any case. 
In the general case (3.29), differentiating equations (3.27), (3.28) and eliminating the variables $\varphi, \xi$, and $\eta$, we obtain the curtailed system (1.7), (1.8), and (1.9), whereas the system (1.11), (2.21), and (2.22) follows from the system (3.22), (3.23), (3.24), (3.25), (3.26), (3.27), and (3.28) directly (i.e., without differentiating). It means that the system (1.11), (2.21), and (2.22) is an integrated system, whereas the curtailed system (1.7), (1.8), and (1.9) is not, although formally they have the same order.

The action of the form (3.21), or close to this form, was obtained by some authors $[18,19]$, but the quantities $g^{\alpha}, \alpha=1,2,3$, are always considered as additional dependent variables (but not as indefinite functions of $\boldsymbol{\xi}$ which can be expressed via initial conditions). The action was not considered as a functional of fixed indefinite functions $g^{\alpha}(\boldsymbol{\xi})$.

Thus, five equations (1.11), (2.21), (2.22) with $S, \rho$, and $\mathbf{v}$, defined, respectively, by (2.18), (2.19), and (2.20), constitute the fifth-order system for five dependent variables $\xi=\left\{\xi_{0}, \boldsymbol{\xi}\right\}, \eta$. Equations (1.7), (1.9), (1.11), (2.21), and (2.22) constitute the seventh-order system for seven variables $\rho, \xi, \varphi, \eta$, and $S$.

If $\Omega^{\alpha \beta} \equiv 0$, it follows from (3.24) that

$$
g^{\alpha}(\boldsymbol{\xi})=\frac{\partial}{\partial \xi_{\alpha}} \Phi(\boldsymbol{\xi}) .
$$

Then it follows from (3.27) and (3.34) that

$$
\mathbf{v}=\frac{\mathbf{j}}{\rho}=\nabla(\varphi+\Phi(\boldsymbol{\xi}))-\eta \nabla S .
$$

In the case of isentropic flow $(\nabla S=0)$, the quantities $\Omega^{\alpha \beta}$ coincide with vorticity, and the fluid flow is irrotational, as it follows from (3.25). In this case, as well as at the fulfillment of (3.35), the dynamic equations (3.23) are satisfied due to relations $\Omega^{\alpha \beta} \equiv 0$, and Lin constraints (1.11) do not follow from dynamic equations (3.23). In this partial case, an addition of the Lin constraints (1.11) to the curtailed system (1.7), (1.9) is not necessary.

The system of equations (3.22), (3.23), (3.24), (3.25), (3.26), (3.27), and (3.28) as well as the system (1.11), (2.18), (2.19), (2.20), (2.21), and (2.22) contain full information on the fluid flow in the infinite space $V$. The system of equations (1.11), (2.21), and (2.22) is a system of five partial differential equations for five dynamic variables $\varphi, \eta$, and $\xi$, and one needs to give initial data for them. But the initial values for variables $\varphi, \eta$, and $\boldsymbol{\xi}$ can be given in the universal form, which is the same for all fluid flows. For instance, one can set

$$
\boldsymbol{\xi}(0, \mathbf{x})=\boldsymbol{\xi}_{\text {in }}(\mathbf{x})=\mathbf{x}, \quad \varphi(0, \mathbf{x})=0, \quad \eta(0, \mathbf{x})=0 .
$$

Then according to (2.18), (2.19), and (2.20), the initial values of variables $\rho, S$, and $\mathbf{v}$ have the form

$$
\begin{aligned}
& \mathbf{v}(0, \mathbf{x})=\mathbf{v}_{\text {in }}(\mathbf{x})=\mathbf{g}(\boldsymbol{\xi})=\mathbf{g}(\mathbf{x}), \\
& \rho(0, \mathbf{x})=\rho_{\text {in }}(\boldsymbol{\xi})=\rho_{\text {in }}(\mathbf{x}), \\
& S(0, \mathbf{x})=S_{\text {in }}(\boldsymbol{\xi})=S_{\text {in }}(\mathbf{x}),
\end{aligned}
$$


where $\rho_{\text {in }}(\mathbf{x}), S_{\text {in }}(\mathbf{x})$, and $\mathbf{v}_{\text {in }}(\mathbf{x})$ are given initial values which determine the fluid flow. Variables $\boldsymbol{\xi}$ label the fluid particles, and a choice of the form of labeling is unessential.

Let now the form of $\varphi(0, \mathbf{x})$ and $\eta(0, \mathbf{x})$ be changed, and we have $\varphi(0, \mathbf{x})=\varphi_{\text {in }}(\mathbf{x})$ and $\eta(0, \mathbf{x})=\eta_{\text {in }}(\mathbf{x})$, where $\varphi_{\text {in }}(\mathbf{x})$ and $\eta_{\text {in }}(\mathbf{x})$ are some given functions. This replacement can be compensated by the change of $\mathbf{g}(\mathbf{x})$ :

$$
\mathbf{g}(\mathbf{x}) \longrightarrow \mathbf{v}_{\text {in }}(\mathbf{x})-\nabla \varphi_{\text {in }}(\mathbf{x})+\eta_{\text {in }}(\mathbf{x}) \nabla S_{\text {in }}(\mathbf{x})
$$

in a way such that the initial values of $\rho, S$, and $\mathbf{v}$ remain the same. All this means that dynamic equations (1.11), (2.18), (2.19), (2.20), (2.21), and (2.22) contain complete (full) information on the fluid flow because the choice of initial values for variables $\varphi, \eta$, and $\boldsymbol{\xi}$ is unimportant for calculation of the fluid flow in the infinite space $V$.

This is valid also for the fluid flow in the space region $x^{3} \geq 0$, as it is shown in [15]. In this case, the boundary conditions for $\mathbf{v}$ at the boundary $x^{3}=0$ are expressed via the arbitrary functions $\mathbf{g}(\boldsymbol{\xi}), \boldsymbol{\xi}=\mathbf{x} \in\left\{\mathbf{x} \mid x^{3}<0\right\}$, whereas the initial conditions are expressed via the arbitrary functions $\mathbf{g}(\boldsymbol{\xi}), \boldsymbol{\xi}=\mathbf{x} \in\left\{\mathbf{x} \mid x^{3} \geq 0\right\}$. Apparently, functions $\mathbf{g}(\mathbf{x})$ determine the initial and boundary conditions for the velocity $\mathbf{v}$ also in the case of the fluid flow in any finite volume $V$, although it is not yet proved.

Thus, the information, which is essential for the fluid flow determination, is described by functions $\rho_{\text {in }}(\mathbf{x}), S_{\text {in }}(\boldsymbol{\xi})$, and $\mathbf{g}$. This information is introduced in dynamic equations in the form of arbitrary functions. Unessential information concerning the methods of the fluid description is given by initial and boundary conditions for variables $\varphi, \eta$, and $\boldsymbol{\xi}$. The variables $\xi, \varphi$, and $\eta$ are auxiliary variables which represent a method of the fluid flow description. The Clebsch potentials $\boldsymbol{\xi}$ label the fluid particles. At the same time, the variables $\boldsymbol{\xi}$ describe displacement of the fluid along their trajectories, and this description does not depend on the method of labeling. Variables $\varphi, \eta$ describe separation of the velocity field $\mathbf{v}$ into parts. The form of this separation is inessential. The auxiliary variables $\xi, \varphi$, and $\eta$ are described by partial differential equations, whereas the fluid flow in itself is described by finite relations containing arbitrary functions $\rho_{\text {in }}(\boldsymbol{\xi}), S_{\text {in }}(\boldsymbol{\xi})$, and $\mathbf{g}(\boldsymbol{\xi})$ (at fixed variables $\varphi, \eta$, and $\boldsymbol{\xi}$, given as functions of $t, \mathbf{x}$ ).

The situation is rather unexpected and unusual. One can obtain a result of calculation of the fluid flow, but it refers to the method of description (variables $\xi, \varphi$, and $\eta$ ), and this method of description is determined by partial differential equations. It is not clear how to resolve and to use this situation effectively.

4. Variational principle for incompressible fluid. Inviscid incompressible fluid of constant density is a special case of the ideal fluid when one may set $\rho=\rho_{0}=$ const. In this case, the continuity equation (3.22), containing time derivative of density $\rho$ and determining time evolution of $\rho$, becomes

$$
\nabla \mathbf{v}=0
$$

Equation (4.1) does not contain time derivatives at all. It is rather a constraint imposed on initial values of velocity $\mathbf{v}$ than a dynamic equation describing evolution of one of dynamic variables. Formally it means that the system of hydrodynamic equations 
ceases to be hyperbolic and becomes elliptic. This circumstance changes the statement of the fluid flow problem.

We set $\rho=\rho_{0}=$ const in the action (3.21) and introduce new variables:

$$
\mathbf{v}=\frac{\mathbf{j}}{\rho_{0}}, \quad \rho_{0}=\text { const }
$$

It is easy to verify that $\eta=\eta(\boldsymbol{\xi})$ and $S=S_{0}(\boldsymbol{\xi})$, and the last term of (3.21) can be incorporated with the term $j^{k} g^{\alpha}(\boldsymbol{\xi}) \partial_{k} \boldsymbol{\xi}_{\alpha}$. Thus, the action for the incompressible fluid looks as follows:

$$
\mathscr{A}_{\mathrm{E}}[\mathbf{v}, \boldsymbol{\xi}, \varphi]=\rho_{0} \int\left\{\frac{\mathbf{v}^{2}}{2}-\mathbf{v} \nabla \varphi-g^{\alpha}(\boldsymbol{\xi}) \partial_{0} \xi_{\alpha}-g^{\alpha}(\boldsymbol{\xi}) \mathbf{v} \nabla \xi_{\alpha}\right\} d t d \mathbf{x},
$$

where $g^{\alpha}(\boldsymbol{\xi})$ are arbitrary fixed functions of $\boldsymbol{\xi}$.

Variation with respect to $\mathbf{v}, \boldsymbol{\xi}$, and $\varphi$ gives

$$
\begin{gathered}
\delta \mathbf{v}: \mathbf{v}=\nabla \varphi+g^{\alpha}(\boldsymbol{\xi}) \nabla \xi_{\alpha}, \\
\rho_{0}^{-1} \frac{\delta A_{\mathrm{E}}}{\delta \xi_{\alpha}}=\Omega^{\alpha \beta}\left(\partial_{0} \xi_{\beta}+\mathbf{v} \nabla \xi_{\beta}\right)=0, \quad \alpha=1,2,3, \\
\rho_{0}^{-1} \frac{\delta \mathscr{A}_{\mathrm{E}}}{\delta \varphi}=\nabla \mathbf{v}=0 .
\end{gathered}
$$

In the general case, condition (3.29) is satisfied, and the multiplier $\Omega^{\alpha \beta}$ in (4.5) may be omitted.

Substituting (4.4) into (4.5) and (4.6), one obtains

$$
\begin{array}{cc}
\Omega^{\alpha \beta}\left(\partial_{0} \xi_{\beta}+\left(\nabla \varphi+g^{\gamma}(\boldsymbol{\xi}) \nabla \xi_{\gamma}\right) \nabla \xi_{\beta}\right)=0, & \alpha=1,2,3, \\
\nabla^{2} \varphi+g^{\alpha, \beta}(\boldsymbol{\xi}) \nabla \xi_{\beta} \nabla \xi_{\alpha}+g^{\alpha}(\boldsymbol{\xi}) \nabla^{2} \xi_{\alpha}=0, & g^{\alpha, \beta} \equiv \frac{\partial g^{\alpha}}{\partial \xi_{\beta}} .
\end{array}
$$

The dynamic equation for $\varphi$ does not contain temporal derivative. If $\Omega^{\alpha \beta} \equiv 0$, the fluid flow is irrotational and dynamic equations (4.7) are fulfilled independently of the Lin constraints which have the form

$$
\partial_{0} \xi_{\beta}+\left(\nabla \varphi+g^{\gamma}(\boldsymbol{\xi}) \nabla \xi_{\gamma}\right) \nabla \xi_{\beta}=0, \quad \alpha=1,2,3 .
$$

Lin constraints (4.9) are not dynamic equations in this case.

Conventional hydrodynamic equations for the incompressible fluid

$$
\nabla \mathbf{v}=0, \quad \partial_{0} \mathbf{v}+(\mathbf{v} \nabla) \mathbf{v}=-\frac{\nabla p}{\rho_{0}}
$$

are obtained from relations (4.4), (4.5), and (4.6). Differentiating (4.4) with respect to $t$, we obtain

$$
\partial_{0} \mathbf{v}=\nabla\left[\partial_{0} \varphi+g^{\alpha}(\boldsymbol{\xi}) \partial_{0} \xi_{\alpha}\right]-\Omega^{\alpha \beta} \partial_{0} \xi_{\beta} \nabla \xi_{\alpha},
$$


where $\Omega^{\alpha \beta}$ is defined by (3.24). It follows from (4.4) that

$$
\mathbf{v} \times(\nabla \times \mathbf{v})=\Omega^{\alpha \beta}(\boldsymbol{\xi}) \nabla \xi_{\beta}(\mathbf{v} \nabla) \xi_{\alpha}
$$

In virtue of (4.5), the last term in the right-hand side of (4.11) coincides with the righthand side of (4.12). Then using identity (3.33), one obtains

$$
\partial_{0} \mathbf{v}+(\mathbf{v} \nabla) \mathbf{v}=\nabla\left(\partial_{0} \varphi+g^{\alpha}(\boldsymbol{\xi}) \partial_{0} \xi_{\alpha}+\frac{1}{2} \mathbf{v}^{2}\right)
$$

Equation (4.13) coincides with the second equation (4.10), provided that we use designation

$$
\frac{p}{\rho_{0}}=\frac{p_{0}}{\rho_{0}}-\frac{1}{2} \mathbf{v}^{2}-\partial_{0} \varphi-g^{\alpha}(\boldsymbol{\xi}) \partial_{0} \xi_{\alpha}, \quad p_{0}=\text { const }
$$

Here the pressure $p$ is determined after the solution of the system of hydrodynamic equations (4.4), (4.5), and (4.6), or (4.10).

We stress that the conventional form (4.10) of hydrodynamic equations is obtained from the hydrodynamic equations (4.4), (4.5), and (4.6) by means of differentiation. It means that the form of hydrodynamic equations (4.4), (4.5), and (4.6) is a result of integration of hydrodynamic equations (4.10) together with the Lin constraints (1.11). It is interesting also that the system of equations (4.4), (4.5), and (4.6) contains time derivatives only in dynamic equations (4.5).

5. Two-dimensional flow of incompressible fluid. We have mentioned in the introduction that the statement of the problem of stream flow is different for the irrotational and rotational cases. This difference appears only after integration. The statement of the problem is different not only for two-dimensional flow of incompressible fluid, but also for any inviscid fluid, and the source of this difference lies in the dynamic equations (3.23) (or (4.5)) which exclude the degrees of freedom connected with rotation in the case $\Omega^{\alpha \beta} \equiv 0$. The method used for derivation of (1.2) cannot be used in general case. So in the following, we present the method in a form which can be expanded to any ideal fluid. The method is based on a use of the GSF (mutual application of the Jacobian technique and description in terms of potentials). To avoid technical complexities, we apply this method to the case of two-dimensional flow of incompressible fluid when the GSF has only one component.

Equations (1.1) and (1.2) are different. The first equation is linear, whereas the second one is quasilinear. The first one can be solved rather easily, whereas the second one can be solved only by means of an iteration procedure. Difference in complexity of (1.1) and (1.2) is technical, whereas the difference in statement of the problem is conceptual. The idea of our investigation is simple. Introducing the GSF $\xi_{2}$, we solve (4.5), (4.6) for any $\xi_{2}$. Then from (4.4), we obtain a dynamic equation for the determination of $\xi_{2}$ and necessary boundary conditions for this equation.

Although it is possible to deal with (4.4), (4.5), and (4.6) for incompressible fluid, we prefer to consider dynamic equations for slightly compressible fluid, whose internal 
energy has the form

$$
E(\rho)=E_{0}\left(\frac{\rho}{\rho_{0}}\right)^{1 / \varepsilon}, \quad E_{0}, \rho_{0}=\text { const }, \varepsilon \ll 1 .
$$

The incompressible fluid appears in the limit $\varepsilon \rightarrow 0$. We consider dynamic equations obtained from the action (3.21) with the entropy $S=$ const and $\eta=0$. These equations have the form

$$
\begin{aligned}
\partial_{k} j^{k} & =0, \quad j^{0} \equiv \rho, \\
\Omega^{\alpha \beta} j^{k} \xi_{\beta, k} & =0, \quad \alpha=1,2,
\end{aligned}
$$

where $\Omega^{\alpha \beta}$ is defined by the relation (3.24):

$$
\begin{gathered}
\frac{j^{\mu}}{\rho}=\partial_{\mu} \varphi+g^{\alpha}(\boldsymbol{\xi}) \xi_{\alpha, \mu}, \quad \mu=1,2, \\
\xi_{\alpha, \mu} \equiv \partial_{\mu} \xi_{\alpha}, \quad \mu, \alpha=1,2, \\
\frac{\partial(\rho E)}{\partial \rho}=-\frac{\mathbf{j}^{2}}{2 \rho^{2}}-\partial_{0} \varphi+g^{\alpha}(\boldsymbol{\xi}) \xi_{\alpha, 0} .
\end{gathered}
$$

Here and in what follows, a summation is produced over repeating Greek indices $(1,2)$ and over repeating Latin indices $(0-2)$. We use designations $\left(x^{0}, x^{1}, x^{2}\right)=(t, x, y)$. The comma before index $k$ denotes differentiation with respect to $x^{k}$, for instance, $\xi_{\alpha, k} \equiv \partial_{k} \xi_{\alpha}$.

We set

$$
J=\frac{\partial\left(\xi_{0}, \xi_{1}, \xi_{2}\right)}{\partial\left(x^{0}, x^{1}, x^{2}\right)}, \quad j^{k}=\frac{\partial J}{\partial \xi_{0, k}}=\frac{\partial\left(x^{k}, \xi_{1}, \xi_{2}\right)}{\partial\left(x^{0}, x^{1}, x^{2}\right)}, \quad k=0,1,2,
$$

where $\xi_{1}, \xi_{2}$ are some functions of $(t, x, y)$. Equations (5.2), (5.3) are satisfied by (5.7) for any functions $\xi_{1}, \xi_{2}$ because of the identities

$$
\partial_{k} \frac{\partial J}{\partial \xi_{0, k}} \equiv 0, \quad \frac{\partial J}{\partial \xi_{0, k}} \xi_{\alpha, k} \equiv 0, \quad \alpha=1,2
$$

Substituting (5.1) in (5.6), we obtain

$$
\left(E_{0}\right)^{\varepsilon}\left(1+\frac{1}{\varepsilon}\right)^{\varepsilon}\left(\frac{\rho}{\rho_{0}}\right)=\left(-\frac{\mathbf{j}^{2}}{2 \rho^{2}}-\partial_{0} \varphi+g^{\alpha}(\boldsymbol{\xi}) \xi_{\alpha, 0}\right)^{\varepsilon} .
$$

In the limit $\varepsilon \rightarrow 0$, (5.9) becomes

$$
\frac{\rho}{\rho_{0}}=\frac{1}{\rho_{0}} \frac{\partial\left(\xi_{1}, \xi_{2}\right)}{\partial\left(x^{1}, x^{2}\right)}=1
$$

In the case of stationary flow, we can assume that $\xi_{2}$ does not depend on the time $t$

$$
\begin{gathered}
\xi_{1}=\xi_{1}(t, x, y), \quad \xi_{2}=\xi_{2}(x, y), \\
\xi_{2,0} \equiv 0 .
\end{gathered}
$$


Equation (5.10) takes the form

$$
\xi_{2,2} \xi_{1,1}-\xi_{2,1} \xi_{1,2}-\rho_{0}=0
$$

We consider (5.13) as an equation for dependent variable $\xi_{1}$ with a given function $\xi_{2}=$ $\xi_{2}(x, y)$. The first-order partial differential equation is equivalent to the system of ordinary equations

$$
\frac{d x}{\xi_{2,2}}=\frac{d y}{-\xi_{2,1}}, \quad \frac{d x}{\xi_{2,2}}=\frac{d \xi_{1}}{\rho_{0}}
$$

It is easy to see that

$$
C_{2}=\xi_{2}(x, y)
$$

is an integral of the first equation of (5.14). Resolving (5.15) with respect to $y$, one obtains

$$
y=F_{2}\left(x, C_{2}\right), \quad \xi_{2}\left(x, F_{2}\left(x, C_{2}\right)\right)=C_{2} .
$$

Substituting (5.16) in the second equation (5.14), one can integrate it in the form

$$
\xi_{1}=\rho_{0}\left[C_{1}\left(t, C_{2}\right)+\int_{-L}^{x} \frac{d x}{\xi_{2,2}\left(x, F_{2}\left(x, C_{2}\right)\right)}\right]_{C_{2}=\xi_{2}(x, y)},
$$

where $C_{1}$ is an arbitrary function of arguments $\left(t, C_{2}\right)$. Here $x=-L$ is the inflow surface $\mathscr{S}_{\text {in }}$, where the inflow conditions are given. The inflow surface is placed in the fluid, and all dynamic equations must be satisfied on $\mathscr{Y}_{\text {in }}$.

Differentiating the second equation (5.16) with respect to $C_{2}$, we obtain

$$
\xi_{2,2}\left(x, F_{2}\left(x, C_{2}\right)\right) F_{2 ; 2}\left(x, C_{2}\right)=1 .
$$

Here and in what follows, the symbol ";" denotes differentiation with respect to one of arguments of the function. Index after symbol ";" denotes the ordinal number of the argument. By means of (5.18), the integral in (5.17) may be written in the form

$$
\int_{-L}^{x} \frac{d x}{\xi_{2,2}\left(x, F_{2}\left(x, C_{2}\right)\right)}=\frac{\partial}{\partial C_{2}} \int_{-L}^{x} F_{2}\left(x, C_{2}\right) d x
$$

The relation (5.17) takes the form

$$
\xi_{1}=\rho_{0}\left[C_{1}\left(t, C_{2}\right)+\frac{\partial}{\partial C_{2}} \int_{-L}^{x} F_{2}\left(x, C_{2}\right) d x\right]_{C_{2}=\xi_{2}(x, y)} .
$$

Differentiating (5.17) with respect to $t$, we obtain

$$
\xi_{1,0}=\rho_{0}\left[\frac{\partial C_{1}\left(t, C_{2}\right)}{\partial t}\right]_{C_{2}=\xi_{2}(x, y)}=\rho_{0} C_{1 ; 1}\left(t, \xi_{2}\right) .
$$


According to (5.11) and (5.7), we have

$$
j^{1}=-\frac{\partial\left(\xi_{1}, \xi_{2}\right)}{\partial\left(x^{0}, x^{2}\right)}=-\xi_{1,0} \xi_{2,2}, \quad j^{2}=\frac{\partial\left(\xi_{1}, \xi_{2}\right)}{\partial\left(x^{0}, x^{1}\right)}=\xi_{1,0} \xi_{2,1},
$$

and (5.4) take the form

$$
\begin{aligned}
& u=v^{1}=-\frac{\xi_{1,0} \xi_{2,2}}{\rho_{0}}=\partial_{1} \varphi+g^{\alpha}(\boldsymbol{\xi}) \xi_{\alpha, 1}, \\
& v=v^{2}=\frac{\xi_{1,0} \xi_{2,1}}{\rho_{0}}=\partial_{2} \varphi+g^{\alpha}(\boldsymbol{\xi}) \xi_{\alpha, 2} .
\end{aligned}
$$

The compatibility condition of (5.23) has the form

$$
-\partial_{2} \frac{\xi_{1,0} \xi_{2,2}}{\rho_{0}}-\partial_{1} \frac{\xi_{1,0} \xi_{2,1}}{\rho_{0}}=\left(g^{\alpha, \beta}(\boldsymbol{\xi})-g^{\beta, \alpha}(\boldsymbol{\xi})\right) \xi_{\alpha, 1} \xi_{\beta, 2}, \quad g^{\alpha, \beta} \equiv \frac{\partial g^{\alpha}}{\partial \xi_{\beta}} .
$$

Substituting (5.21) and using designation (3.24), we obtain

$$
\psi_{11}+\psi_{22}=-\rho_{0} \Omega^{12}\left(\xi_{1}, \xi_{2}\right),
$$

where the stream function $\psi$ is defined by the relation

$$
\psi=C_{0}\left(t, \xi_{2}\right)=\int C_{1 ; 1}\left(t, \xi_{2}\right) d \xi_{2} .
$$

Relation (5.26) is chosen in such a way that (5.23) coincide with (1.4). We resolve relation (5.26) with respect to $\xi_{2}$ in the form $\xi_{2}=Q(t, \psi)$, where $Q(t, \psi)$ is the functional inverse of $C_{0}\left(t, \xi_{2}\right)$

$$
C_{0}(t, Q(t, \psi))=\psi
$$

Substituting $\xi_{2}$ in (5.17), we obtain Clebsch potentials $\xi_{1}$ and $\xi_{2}$ expressed via $\psi$ by means of relations

$$
\xi_{2}=Q(t, \psi), \quad \xi_{1}=\xi_{1}(t, x, \psi)=\rho_{0}\left[C_{1}\left(t, C_{2}\right)+\frac{\partial}{\partial C_{2}} \int_{-L}^{x} F_{2}\left(x, C_{2}\right) d x\right]_{C_{2}=Q(t, \psi)} .
$$

According to (5.22), (5.21), and (5.26), we obtain

$$
j^{1}=-\rho_{0} \psi_{2}, \quad j^{2}=\rho_{0} \psi_{1}
$$

Dynamic equation (5.25) for the stream function $\psi$ is the second-order partial differential equation of elliptic type. It contains indefinite functions $\Omega^{12}\left(\xi_{1}, \xi_{2}\right), C_{0}\left(t, \xi_{2}\right)$ which are determined from the inflow conditions. The time $t$ appears in dynamic equation only as a parameter describing evolution of the Clebsch potential $\xi_{1}$. The dynamic equation (5.25) is applicable for arbitrary two-dimensional flow of ideal incompressible fluid. 
REMARK 5.1. Equation (5.25) can be obtained by direct integration of Euler equations for two-dimensional flow of incompressible fluid. Indeed, eliminating pressure from (4.10) for two-dimensional flow, we obtain

$$
u_{1}+v_{2}=0, \quad \partial_{2}\left(u_{0}+u u_{1}+v u_{2}\right)=\partial_{1}\left(v_{0}+u v_{1}+v v_{2}\right)
$$

where $u$ and $v$ are velocity components along $x$-axis and $y$-axis, respectively. Introducing the stream function $\psi$,

$$
u=-\psi_{2}, \quad v=\psi_{1},
$$

we satisfy the first equation of (5.30) identically. The second equation of (5.30) takes the form

$$
\partial_{0} \omega-\frac{\partial(\omega, \psi)}{\partial(x, y)}=0, \quad \omega \equiv \psi_{11}+\psi_{22}
$$

In the stationary case, the result of integration is considered in the introduction.

In the case of arbitrary flow, (5.32) is reduced to the form

$$
\frac{d \omega}{d t}=\partial_{0} \omega+u \partial_{1} \omega+v \partial_{2} \omega=0 .
$$

Solution of (5.33) can be presented in the form

$$
\omega=\psi_{11}+\psi_{22}=\Omega\left(\xi_{1}, \xi_{2}\right)
$$

where $\xi_{1}$ and $\xi_{2}$ are two independent solutions of (5.33), that is, $\xi_{1}$ and $\xi_{2}$ are Clebsch potentials satisfying (1.11). Thus, the problem of the Euler system integration appears to be connected with consideration of the Lin constraints. The statement that we can investigate the fluid motion, considering only the Euler system of dynamic equations and ignoring completely Lin constraints, is valid only in the case $\Omega \equiv 0$. In the general case, it is only an illusion. In reality, the Lin constraints are hidden inside the Euler system and appear after integration.

In the simplest case, when all fluid particles cross boundary, and there are no closed stream lines, the function $\Omega\left(\xi_{1}, \xi_{2}\right)$ is determined from the inflow conditions on the boundary of the considered flow. If the flow contains fluid particles which move all the time inside the considered volume and do not cross its boundary, the function $\Omega\left(\xi_{1}, \xi_{2}\right)$ is to be given for these particles also, although such an assignment is impossible via boundary conditions. Here we will consider only the simplest case of rotational flow when there are no closed stream lines. The case with closed stream lines of rotational flow inside the irrotational flow is considered in [9]. In this paper, the function $\Omega\left(\xi_{2}\right)=$ $f(\psi)$ is given on the closed stream lines without a reference to boundary conditions.

In the considered case, the dynamic equation (5.34) appears to contain information on boundary conditions. It means that, studying rotational nonstationary flows, a separate investigation of dynamic equations and boundary conditions becomes impossible. Appearance of several indefinite functions of $\psi$ in dynamic equations of type (1.2) for stationary rotational flow is well known (see, for instance, survey [14]). Unfortunately, an investigation of the origin of these functions is sometimes absent. 
Obtaining the basic equation (5.25), we used properties of Clebsch potentials described by (5.4). These relations contain arbitrary functions $g^{\alpha}(\boldsymbol{\xi})$, which describe influence of boundary conditions on the form of dynamic equations. Indefinite functions $g^{\alpha}(\xi)$ describe general properties of the ideal fluid, which could be applied not only for the investigation of incompressible fluid. Information contained in three functions $g^{a}(\boldsymbol{\xi})$ of three arguments $\xi_{1}, \xi_{2}$, and $\xi_{3}$ is much more than information contained in one function $\Omega\left(\xi_{1}, \xi_{2}\right)$ of two arguments. Maybe, not all this information is essential, but some part of this information is essential for sure. It is the reason why we investigate dynamic equations (4.4), (4.5), and (4.6), but not Euler system (5.30), which contain this information only in implicit form.

The last shorter derivation of (5.25) in Remark 5.1 ignores general relations (5.3), (5.4), but nevertheless, finally it leads to (5.34), that is, to a description in terms of Clebsch potentials.

6. Inflow conditions for stationary flow. We suppose that the stationary inflow conditions are given at the plane $\mathscr{S}_{\text {in }}: x=-L=$ const. (We consider the case when there are no closed stream lines inside the considered volume.) The inflow surface $\mathscr{S}_{\text {in }}$ lies in the fluid completely, and all dynamic equations are satisfied on $\mathscr{S}_{\text {in }}$. In the stationary case, the stream function $\psi$ does not depend on time $t$. One may set in (5.26) $C_{0}\left(t, \xi_{2}\right)=C_{0}\left(\xi_{2}\right)$. On the inflow surface, we choose the function $\xi_{2}$ in the form

$$
\xi_{2}(-L, y)=y
$$

According to (5.21) and (5.22), the inflow conditions have the form

$$
\begin{gathered}
u(-L, y)=-C_{1 ; 1}(-L, y)=V_{x}(y), \\
\frac{\partial v}{\partial x}(-L, y)=\frac{1}{\rho_{0}}\left[\partial_{1}\left(\xi_{1,0}(t, y) \xi_{2,1}(x, y)\right)\right]_{x=-L}=W(y),
\end{gathered}
$$

where $u$ and $v$ are velocity components along $x$-axis and $y$-axis, respectively, and $V_{x}(y)$ and $W(y)$ are supposed to be known functions determining inflow conditions of the fluid. We obtain from (6.2) and (5.26)

$$
C_{1}(t, y)=-\int_{0}^{t} V_{x}(y) d t \equiv S(y) t, \quad C_{0}(y)=-\int V_{x}(y) d y .
$$

The left-hand side of (5.24) at $x=-L$ is equal to

$$
\frac{\partial}{\partial y} u(-L, y)-\frac{\partial v}{\partial x}(-L, y)=\frac{\partial}{\partial y} V_{x}(y)-W(y)
$$

whereas the right-hand side of (5.24) at $x=-L$ is equal to $\rho_{0} \Omega^{12}\left(\xi_{1}(t,-L, y), y\right)$. Then using representation (5.17), we obtain from (5.24) and (5.28) at $x=-L$

$$
\rho_{0} \Omega^{12}\left(\rho_{0} C_{1}(t, y), y\right)=\frac{\partial}{\partial y} V_{x}(y)-W(y) \equiv U(y),
$$

where $U(y)$ is a known function of variables $y$. As far as the right-hand side of (6.6) does not depend on $t$, the left-hand side of (6.6) does not depend on $C_{1}$, and $\Omega^{12}$ depends 
only on $\xi_{2}$ :

$$
\Omega^{12}\left(\xi_{1}, \xi_{2}\right)=\Omega^{12}\left(0, \xi_{2}\right)=\frac{1}{\rho_{0}} U\left(\xi_{2}\right)=\frac{1}{\rho_{0}}\left(\frac{\partial}{\partial \xi_{2}} V_{x}\left(\xi_{2}\right)-W\left(\xi_{2}\right)\right) .
$$

Thus, inflow conditions (6.2), (6.3) admit one to determine indefinite functions $\Omega^{12}$ and $C_{1}$. It is interesting that inflow conditions are given at the inflow surface $\mathscr{Y}_{\text {in }}: x=-L$ by the velocity component $u$ and by derivative $\partial v / \partial x$ (normal to $\mathscr{Y}_{\text {in }}$ ) of the component $v$ (but not by the component $v$ itself). Information given by the velocity component $v$ on the inflow surface $\mathscr{Y}_{\text {in }}$ is insufficient for the determination of indefinite functions $\Omega^{12}$ and $C_{1}$. The derivative $\partial v / \partial x$ together with $\partial u / \partial y$ determine vorticity. The derivative $\partial u / \partial y$ can be calculated if the velocity $u$ is given on the inflow surface, whereas $\partial v / \partial x$ cannot. To determine vorticity, we need to give $\partial v / \partial x$ on the inflow surface, whereas the tangent component $v$ itself appears to be unnecessary for the vorticity determination.

7. Problem of stationary flow over obstacle. We consider a flow of incompressible fluid inside the space region $V$ bounded by two walls $\mathscr{Y}_{1}: y=-R=$ const and $\mathscr{I}_{2}: y=R$, by inflow surface $\mathscr{Y}_{\text {in }}: x=-L=$ const, outflow surface $\mathscr{Y}_{\text {out }}: x=L$, and by the surface $\mathscr{S}_{\mathrm{ob}}$ of some obstacle, placed in vicinity of the coordinate origin. The shape of the obstacle is determined by the relation

$$
\mathscr{Y}_{\mathrm{ob}}: \Sigma_{\mathrm{ob}}(x, y)=0
$$

The boundary conditions are formulated as follows:

$$
\begin{gathered}
\mathscr{S}_{1}: \psi(x,-R)=\psi_{1}=\text { const, } \quad \mathscr{S}_{2}: \psi(x, R)=\psi_{2}=\text { const, } \\
\mathscr{S}_{\mathrm{ob}}:\left.\psi(x, y)\right|_{x, y \in \sum_{\mathrm{ob}}}=\psi_{\mathrm{ob}}=\text { const. }
\end{gathered}
$$

Constants $\psi_{1}$ and $\psi_{2}$ are connected by the relation

$$
\psi_{2}-\psi_{1}=-\int_{-R}^{R} V_{x}(y) d y .
$$

Conditions (7.2) mean that the normal velocity component vanishes on all walls. The value of $\psi_{\mathrm{ob}}$ describes a circulation around the obstacle because $\psi_{\mathrm{ob}}-\psi_{1}$ describes the mean value of the flux between $\mathscr{Y}_{1}$ and $\mathscr{S}_{\mathrm{ob}}$. For instance, if $\psi_{\mathrm{ob}}=\psi_{1}$, the circulation around the obstacle is such that the fluid flows only between $\mathscr{S}_{2}$ and $\mathscr{S}_{\mathrm{ob}}$.

Boundary condition on the inflow surface $\mathscr{S}_{\text {in }}$ has the form

$$
\mathscr{S}_{\text {in }}: \psi(-L, y)=\psi_{1}-\int_{-R}^{y} V_{x}(y) d y,
$$

where the velocity component $V_{x}(y)$ along the $x$-axis is presumed to be given. The boundary condition on the outflow surface $\mathscr{S}_{\text {out }}$ has the form

$$
\mathscr{S}_{\text {out }}: \psi(L, y)=\psi_{\text {out }}(y), \quad \psi_{\text {out }}(-R)=\psi_{1}, \quad \psi_{\text {out }}(R)=\psi_{2},
$$

where $\psi_{\text {out }}(y)$ is some given function. It describes the velocity distribution on the outflow surface. To understand why such a distribution should be given and why it 
is not determined by the inflow condition, we imagine that outside the volume $V$ in vicinity of $\mathscr{Y}_{\text {out }}$, there are some obstacles. Distribution of velocity on $\mathscr{S}_{\text {out }}$ depends on size, shape, and position of these obstacles. Description of these obstacles is carried out by $\psi_{\text {out }}(y)$.

We consider a flow over the obstacle and rewrite dynamic equation (5.25) in the form

$$
\begin{gathered}
\mathscr{L} \psi=\Omega(\psi), \\
\mathscr{L} \psi=\nabla^{2} \psi=\psi_{11}+\psi_{22}, \\
\Omega(\psi)=-\rho_{0} \Omega^{12}\left(\xi_{2}\right)=-U\left(\xi_{2}\right)=-U(Q(\psi)),
\end{gathered}
$$

where the function $U$ is defined as a function of argument $y$ by means of the relation (6.6):

$$
U(y)=\frac{\partial}{\partial y} V_{x}(y)-W(y)
$$

Function $\xi_{2}=Q(\psi)$ is defined as a function of argument $\psi$ by means of the relation (7.4):

$$
\psi=\psi_{1}-\int_{-R}^{Q(\psi)} V_{x}(y) d y
$$

In particular, if $V_{x}(y)=V_{0}=$ const, $\xi_{2}=Q(\psi)=\left(\psi_{1}-\psi\right) / V_{0}$.

Dynamic equation (7.6) is a nonlinear equation in general. Its solution is difficult. The goal of this paper is to provide a statement of the stream flow problem because even this problem is not solved for irrotational flows. So far as the solution of (7.6) is concerned, it is a special mathematical problem which can be solved by different methods.

If vorticity $\Omega$ is small, dynamic equation (7.6) can be solved by means of an iteration procedure. To obtain the zeroth-order approximation $\psi_{(0)}$, we consider linear equation

$$
\mathscr{L} \psi_{(0)}=\nabla^{2} \psi_{(0)}=0
$$

with boundary conditions (7.2), (7.3), (7.4), and (7.5), which is written for brevity in the form

$$
\left[\psi_{(0)}\right]_{\Sigma}=\psi_{\Sigma}(t, \mathbf{x}), \quad \mathbf{x} \in \Sigma,
$$

where $\Sigma$ is the boundary of the volume filled by fluid.

Let $G\left(\mathbf{x} \mid \mathbf{x}^{\prime}\right)$ be the Green function for (7.11). It is defined by the relations

$$
\nabla^{2} G\left(\mathbf{x} \mid \mathbf{x}^{\prime}\right)=-4 \pi \delta\left(\mathbf{x}-\mathbf{x}^{\prime}\right), \quad G\left(\mathbf{x} \mid \mathbf{x}^{\prime}\right)_{x \in \Sigma}=0 .
$$

Solution of (7.11) is given by the formula

$$
\psi_{(0)}(\mathbf{x})=-\frac{1}{4 \pi} \int_{\Sigma} \frac{\partial G\left(\mathbf{x} \mid \mathbf{x}^{\prime}\right)}{\partial n^{\prime}} \psi_{\Sigma}\left(x^{\prime}\right) d S^{\prime},
$$


where $\partial / \partial n^{\prime}$ means a derivative with respect to $x^{\prime}$ in the direction normal to $\Sigma$. Solution (7.14) is an irrotational approximation to the solution of (7.6) for the rotational flow.

The first approximation is determined by the relation

$$
\psi_{(1)}(\mathbf{x})=\psi_{(0)}(\mathbf{x})-\frac{1}{4 \pi} \int_{V} G\left(\mathbf{x} \mid \mathbf{x}^{\prime}\right) \Omega\left(\psi_{(0)}\left(\mathbf{x}^{\prime}\right)\right) d V^{\prime},
$$

where integration is produced over the volume $V$ filled with the fluid:

$$
\psi_{(n)}(\mathbf{x})=\psi_{(0)}(\mathbf{x})-\frac{1}{4 \pi} \int_{V} G\left(\mathbf{x} \mid \mathbf{x}^{\prime}\right) \Omega\left(\psi_{(n-1)}\left(\mathbf{x}^{\prime}\right)\right) d V^{\prime}, \quad n=2,3, \ldots
$$

If the iteration process converges, we obtain a solution of the problem of rotational stationary flow around an obstacle. One can hope that the iteration process appears to be effective at least for small vorticity $\Omega$.

8. Examples of two-dimensional stationary flow. We consider a stationary flow bounded by two parallel plane walls $\mathscr{S}_{1}: y=0$ and $\mathscr{S}_{2}: y=b$, with inflow and outflow surfaces $\mathscr{Y}_{\text {in }}: x=0$ and $\mathscr{Y}_{\text {out }}: x=a$. To obtain linear equation (7.6) which has a simple exact solution, we choose the following boundary conditions:

$$
\begin{gathered}
\psi(x, 0)=0, \quad \psi(x, b)=0, \\
\psi(0, y)=V_{0} \sin \frac{\pi y}{b}, \quad \psi(a, y)=V_{0} \sin \frac{\pi y}{b}, \quad V_{0}=\text { const } \\
\psi_{11}(0, y)=k^{2} V_{0} \sin \frac{\pi y}{b}, \quad k=\sqrt{\Omega+\left(\frac{\pi}{b}\right)^{2}}, \Omega=\text { const. }
\end{gathered}
$$

In this case, the vorticity $\Omega(\psi)$ in the right-hand side of (7.6) on the boundary $x=0$ is determined by the first condition (8.2) and by (8.3):

$$
\begin{aligned}
\frac{\partial}{\partial y} u(0, y)-\frac{\partial}{\partial x} v(0, y) & =\psi_{11}(0, y)+\psi_{22}(0, y) \\
& =\left(k^{2}-\left(\frac{\pi}{b}\right)^{2}\right) V_{0} \sin \frac{\pi y}{b} \\
& =\Omega \psi(0, y) .
\end{aligned}
$$

It means that the function $\Omega(\psi)$ in the right-hand side of (7.6) has the form $\Omega(\psi)=\Omega \psi$, $\Omega=$ const, and the dynamic equation takes the form of linear equation

$$
\nabla^{2} \psi=\Omega \psi, \quad \Omega=\text { const. }
$$

Its solution, satisfying the boundary conditions (8.1), (8.2), has the form

$$
\psi=V_{0} \frac{\sinh (k x)+\sinh (k(a-x))}{\sinh (k a)} \sin \frac{\pi y}{b}, \quad k=\sqrt{\Omega+\left(\frac{\pi}{b}\right)^{2}} .
$$

This solution describes a set of flows with vanishing total flux through the inflow surface and different vorticities $\Omega$. Flows of this set are distinguished by the power of circulation which is described by the parameter $\Omega$. 
We consider two-dimensional stationary slightly rotational flow between two concentric circular cylinders of radii $a$ and $b(a<b)$. We choose the coordinate system with the $z$-axis directed along the cylinder axis and coordinate origin on the cylinder axis. The $x$-axis is directed along the mean velocity $V_{0}$ of the flow. We introduce polar coordinates $(r, \varphi)$ in the $(x, y)$-plane and set the boundary conditions for the stream function as follows:

$$
\begin{gathered}
\psi(a, \varphi)=0, \quad \psi(b, \varphi)=-V_{0}\left(1-\frac{a^{2}}{b^{2}}\right) b \sin \varphi, \\
{\left[\frac{1}{r} \frac{\partial}{\partial r}\left(r \frac{\partial \psi(r, \varphi)}{\partial r}\right)\right]_{r=b}=\omega_{1}(\varphi)=\omega(\psi(b, \varphi))= \begin{cases}\omega(\psi) & \text { if }|\psi|<\psi_{0}, \\
0 & \text { if }|\psi|>\psi_{0},\end{cases} }
\end{gathered}
$$

where $\omega_{1}(\varphi)$ is some given function of $\varphi$. It is supposed that the flow is rotational only in the part of fluid which flows near the cylinder. Let $-\psi_{0}$ and $\psi_{0}$,

$$
\psi_{0}=V_{0}\left(1-\frac{a^{2}}{b^{2}}\right) N a
$$

be values of the stream function on the boundary between the rotational and irrotational parts of the flow; $N a$ is the transverse size of the rotational region $(N \gg 1)$. After the statement of the problem, we set $b \rightarrow \infty$. The irrotational flow of the zeroth approximation around the cylinder is described by the stream function $\psi_{(0)}$ which is given by the expression [12]:

$$
\psi_{(0)}(r, \varphi)=-V_{0}\left(1-\frac{a^{2}}{r^{2}}\right) r \sin \varphi, \quad r>a
$$

where $r, \varphi$ are polar coordinates on the $(x, y)$-plane:

$$
x=r \cos \varphi, \quad y=r \sin \varphi .
$$

The irrotational flow (8.10) is used at formulation of boundary conditions on the external cylinder of radius $b$.

According to (7.15) and (8.7), (8.8), (8.9), and (8.10), we obtain for the first approximation

$$
\psi_{(1)}(r, \varphi)=\psi_{(0)}(r, \varphi)-\frac{1}{4 \pi} \iint_{\left|\psi_{(0)}\left(r^{\prime}, \varphi^{\prime}\right)\right|<\psi_{0}} G\left(r, \varphi \mid r^{\prime}, \varphi^{\prime}\right) \omega\left(\psi_{(0)}\left(r^{\prime}, \varphi^{\prime}\right)\right) r^{\prime} d r^{\prime} d \varphi^{\prime},
$$

where $\omega(\psi)$ is defined by the relation (8.8), and the Green function $G\left(r, \varphi \mid r^{\prime}, \varphi^{\prime}\right)$ is taken for the case $b=\infty$. It has the form

$$
G\left(r, \varphi \mid r^{\prime}, \varphi^{\prime}\right)=\ln \frac{a^{2}+r^{2} r^{\prime 2} / a^{2}-2 r r^{\prime} \cos \left(\varphi-\varphi^{\prime}\right)}{r^{2}+r^{\prime 2}-2 r r^{\prime} \cos \left(\varphi-\varphi^{\prime}\right)},
$$


and the integration is produced over the region, where $\left|\psi_{(0)}(r, \varphi)\right|<\psi_{0}=$ const. According to (8.9), (8.10), this region is defined by the relation

$$
\left|V_{0}\left(1-\frac{a^{2}}{r^{2}}\right) r \sin \varphi\right|<V_{0} N a, \quad r>a, b=\infty,
$$

where $N \gg 1$. If

$$
r<R=a\left(\frac{N}{2}+\sqrt{\left(\frac{N}{2}\right)^{2}+1}\right)
$$

inequality (8.14) is satisfied for any angle $\varphi$, and for $r^{\prime}<R$, one should integrate in (8.12) over all values of angle $\varphi^{\prime}$. For $r^{\prime}>R$, it is useful to introduce the variable $\eta$ by means of relation

$$
\varphi^{\prime}=\Phi(r, \eta)=\arcsin \frac{\eta}{V_{0}\left(1-a^{2} / r^{2}\right) r}
$$

and integrate over $\eta$ in the region, where $r^{\prime}>R$.

We obtain

$$
\psi_{(1)}=\psi_{(0)}+I_{1}+I_{2}+I_{3},
$$

where

$$
\begin{aligned}
& I_{1}=-\frac{1}{4 \pi} \int_{a}^{R} r^{\prime} d r^{\prime} \int_{0}^{2 \pi} G\left(r, \varphi \mid r^{\prime}, \varphi^{\prime}\right) \omega\left(\psi_{(0)}\left(r^{\prime}, \varphi^{\prime}\right)\right) d \varphi^{\prime}, \\
& I_{2}=\frac{1}{4 \pi} \int_{R}^{\infty} r^{\prime 2} d r^{\prime} \int_{-\Phi\left(r^{\prime}, \psi_{0}\right)}^{\Phi\left(r^{\prime}, \psi_{0}\right)} \frac{G\left(r, \varphi \mid r^{\prime}, \Phi\left(r^{\prime}, \eta\right)\right) \omega(-\eta) V_{0}\left(1-a^{2} / r^{\prime 2}\right) d \eta}{\sqrt{V_{0}^{2}\left(1-a^{2} / r^{\prime 2}\right)^{2} r^{\prime 2}-\eta^{2}}}, \\
& I_{3}=\frac{1}{4 \pi} \int_{R}^{\infty} r^{\prime 2} d r^{\prime} \int_{\pi-\Phi\left(r^{\prime}, \psi_{0}\right)}^{\pi+\Phi\left(r^{\prime}, \psi_{0}\right)} \frac{G\left(r, \varphi \mid r^{\prime}, \Phi\left(r^{\prime}, \eta\right)\right) \omega(-\eta) V_{0}\left(1-a^{2} / r^{\prime 2}\right) d \eta}{\sqrt{V_{0}^{2}\left(1-a^{2} / r^{\prime 2}\right)^{2} r^{\prime 2}-\eta^{2}}},
\end{aligned}
$$

where $\psi_{0}=V_{0} N a$. Although calculations of integrals (8.18) is not simple, they give the first approximation of the rotational flow around the cylinder.

9. Conclusion. Theory of rotational flows is more complicated than that of irrotational flows. There are two reasons for this.

(1) Dynamic equations for rotational flows appear to be nonlinear almost always. They cannot be solved exactly.

(2) The problem of rotational stream flow is not yet stated properly.

The first problem is pure mathematical. Existence of this problem is quite clear for all researchers. The second problem is physical. We believe that hardly anybody of researchers guesses on existence of this problem. The first problem is very difficult. 
The second problem is not so difficult, but it is much more important than the first problem. Why?

First, because existence of the second problem in itself seems to be problematic. Second, the irrotational flow is a special degenerate case of the rotational flow, when vorticity vanishes. Transition from the general case to the degenerate one is rather simple. The reverse transition from the degenerate case to the general one is much more difficult because the general case contains additional information which is absent in the degenerate case. If we could not evaluate correctly this additional information, we could not construct a perfect theory. Practically, we ignore dynamic equations (1.11) and boundary conditions for determination of vorticity. As a result we obtain imperfect theory, where some degrees of freedom are ignored. In some cases, these hidden degrees of freedom manifest themselves suddenly, and we try to understand what could be meant by this effect. At any rate, a serious mathematical investigation of such difficult hydrodynamical problems as turbulence is impossible if the underlying rotational flow theory is incomplete.

\section{REFERENCES}

[1] V. L. Berdichevskiĭ, Variational Principles of Continuum Mechanics, Nauka, Moscow, 1983.

[2] F. P. Bretherton, A note on Hamilton's principle for perfect fluids, J. Fluid Mech. 44 (1970), 19-31.

[3] M. G. Calkin, An action principle for magnetohydrodynamics, Canad. J. Phys. 41 (1963), 2241-2251.

[4] A. Clebsch, Über eine allgemaine transformation der hydrodynamischen Gleichungen, J. reine angew. Math. 54 (1857), 293-312 (German).

[5] _ Über die integration der hydrodynamischen Gleichungen, J. reine angew. Math. 56 (1859), 1-10 (German).

[6] B. Davydov, Variational principle and canonical equations for an ideal fluid, Dokl. Akad. Nauk SSSR 69 (1949), 165-168 (Russian).

[7] C. Eckart, The electrodynamics of material media, Phys. Rev. (2) 54 (1938), 920-923.

[8] _ Variation principles of hydrodynamics, Phys. Fluids 3 (1960), 421-427.

[9] A. Elcrat, B. Fornberg, and K. Miller, Some steady axisymmetric vortex flows past a sphere, J. Fluid Mech. 433 (2001), 315-328.

[10] J. L. Friedman and B. F. Schutz, Lagrangian perturbation theory of nonrelativistic fluids, Astrophys. J. 221 (1978), no. 3, part 1, 937-957.

[11] J. W. Herivel, The derivation of the equations of motion of an ideal fluid by Hamilton's principle, Math. Proc. Cambridge Philos. Soc. 51 (1955), 344-349.

[12] H. Lamb, Hydrodynamics, Dover, New York, 1932.

[13] C. C. Lin, Hydrodynamics of helium II, Proc. Int. Sch Phys. Course XXI, Academic, New York, 1963, pp. 93-146.

[14] R. R. Long, Finite amplitude disturbances in the flow of inviscid rotating and stratified fluid over obstacles, Ann. Rev. Fluid Mech. 4 (1972), 69-92.

[15] Yu. A. Rylov, Integration of complete system of dynamic equations for ideal fluid, preprint, 1999, http://arxiv.org/abs/physics/9905044.

[16] _ Spin and wave function as attributes of ideal fluid, J. Math. Phys. 40 (1999), no. 1, 256-278.

[17] R. Salmon, Hamilton's principle and Ertel's theorem, Mathematical Methods in Hydrodynamics and Integrability in Dynamical Systems (La Jolla, Calif, 1981), AIP Conf. Proc., vol. 88, Amer. Inst. Phys., New York, 1982, pp. 127-135. , Hamilton fluid mechanics, Ann. Rev. Fluid Mech. 20 (1988), 225-256. 
[19] R. L. Seliger and F. R. S. Whitham, Variational principles in continuum mechanics, Proc. Roy. Soc. London Ser. A 305 (1968), 1-25.

[20] V. E. Zakharov and E. A. Kuznetsov, Hamilton formalizm for systems of the hydrodynamic type, Uspehi Fiz. Nauk 167 (1997), 1137-1167 (Russian).

Yuri A. Rylov: Institute for Problems in Mechanics, Russian Academy of Sciences, 101-1 Vernadskii Avenue, Moscow 119526, Russia

E-mail address: rylov@ipmnet.ru 


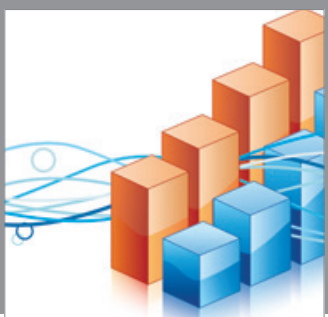

Advances in

Operations Research

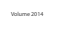

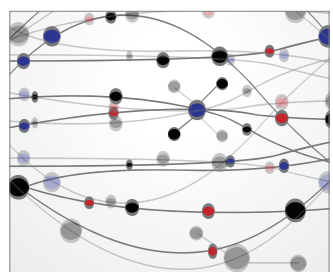

\section{The Scientific} World Journal
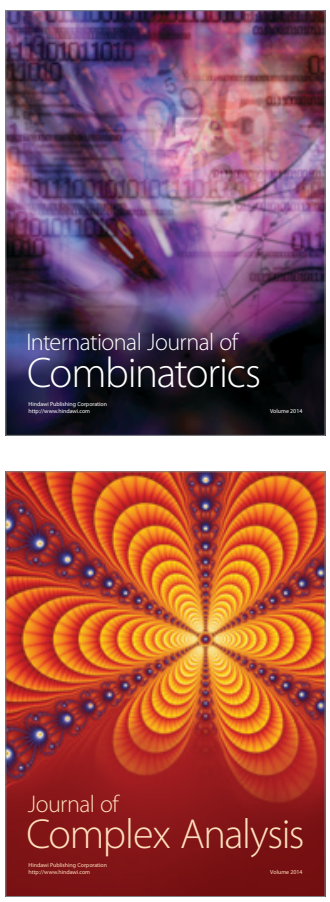

International Journal of

Mathematics and

Mathematical

Sciences
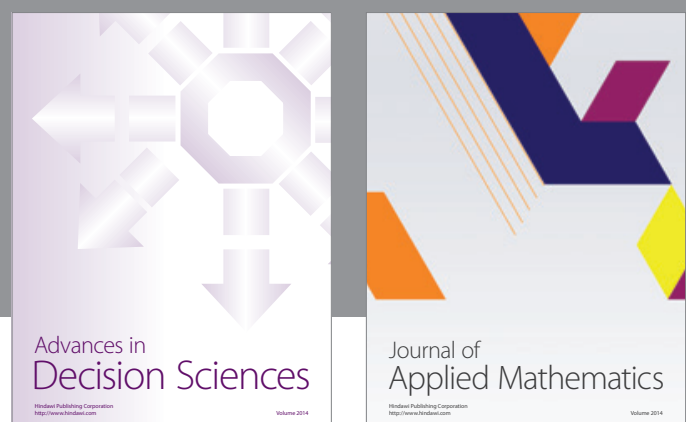

Journal of

Applied Mathematics
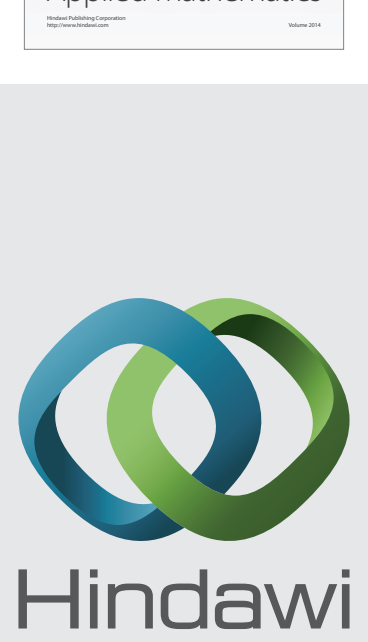

Submit your manuscripts at http://www.hindawi.com
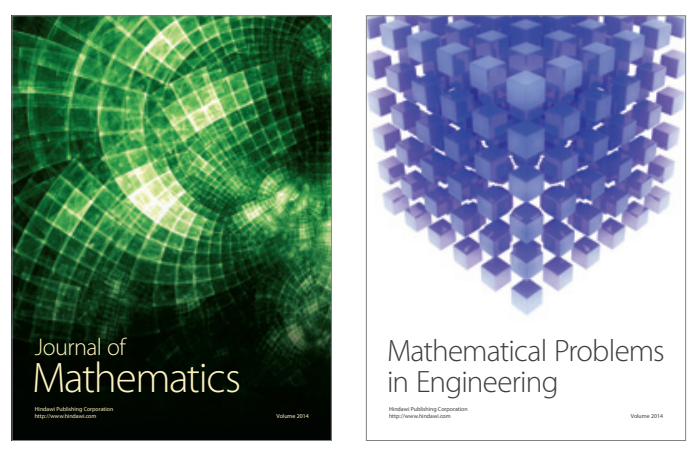

Mathematical Problems in Engineering
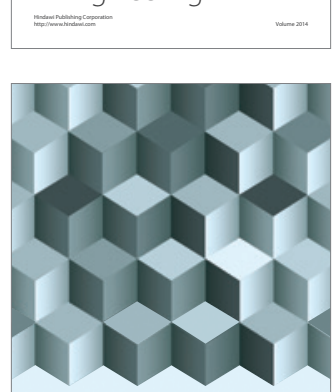

Journal of

Function Spaces
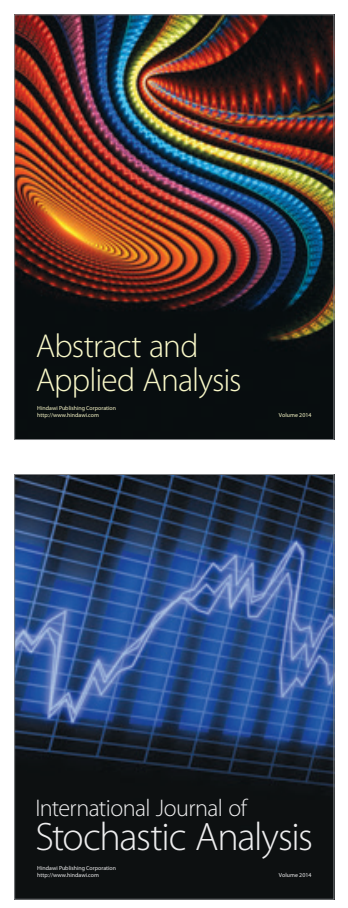

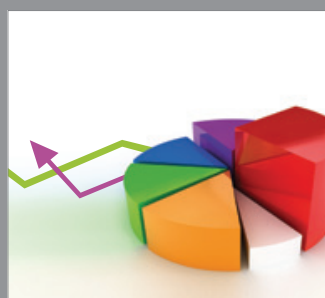

ournal of

Probability and Statistics

Promensencen
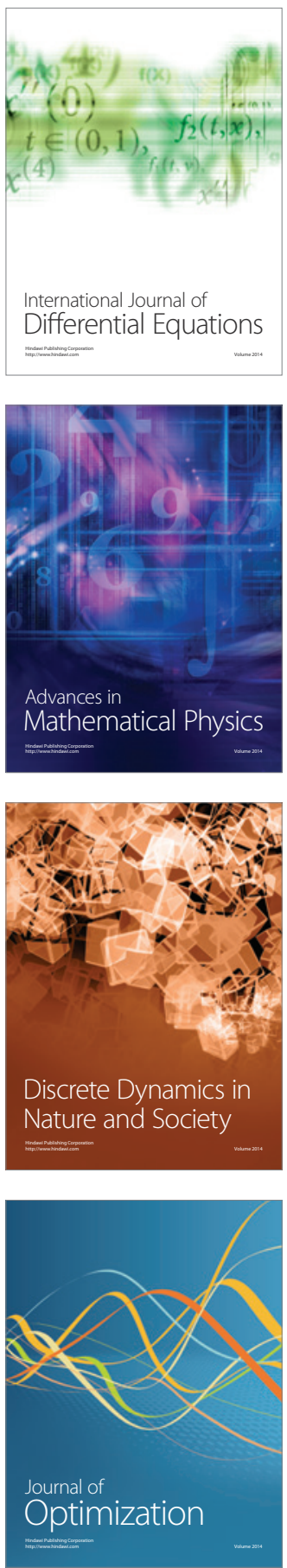